\title{
Spatially Correlated Content Caching for Device-to-Device Communications
}

\author{
Derya Malak, Mazin Al-Shalash, and Jeffrey G. Andrews
}

\begin{abstract}
We study optimal geographic content placement for device-to-device (D2D) networks in which each file's popularity follows the Zipf distribution. The locations of the D2D users (caches) are modeled by a Poisson point process (PPP) and have limited communication range and finite storage. Inspired by the Matérn hard-core (type II) point process that captures pairwise interactions between nodes, we devise a novel spatially correlated caching strategy called hard-core placement (HCP) such that the D2D nodes caching the same file are never closer to each other than the exclusion radius. The exclusion radius plays the role of a substitute for caching probability. We derive and optimize the exclusion radii to maximize the hit probability, which is the probability that a given D2D node can find a desired file at another node's cache within its communication range. Contrasting it with independent content placement, which is used in most prior work, our HCP strategy often yields a significantly higher cache hit probability. We further demonstrate that the HCP strategy is effective for small cache sizes and a small communication radius, which are likely conditions for D2D.
\end{abstract}

\section{INTRODUCTION}

D2D communication is a promising technique for enabling proximity-based applications and increased offloading from the heavily loaded cellular network, and is being actively standardized by 3GPP [2]. The efficacy of D2D caching networks relies on users possessing content that a nearby user wants. Therefore, intelligent caching of popular files is critical for D2D to be successful. Caching has been shown to provide increased spectral reuse and throughput gain in D2D-enabled networks [3], and the optimal way to cache content is studied from different perspectives, e.g. using probabilistic placement [4], maximizing cache-aided spatial throughput

This work in part appeared in Proc. IEEE Intl. Symposium on Info. Theory, Barcelona, Spain, July 2016 [1].

D. Malak and J. G. Andrews are with the Wireless Networking and Communications Group (WNCG), The University of Texas at Austin, Austin, TX 78701 USA (email: deryamalak@utexas.edu; jandrews@ece.utexas.edu). M. Al-Shalash is with Huawei Technologies, Plano, TX 75075 USA (e-mail: mshalash@huawei.com). Last revised: October 4, 2017. 
[5], but several aspects of optimal caching exploiting spatial correlations for network settings have not been explored. Intuitively, given a finite amount of storage at each node, popular content should be seeded into the network in a way that maximizes the hit probability that a given D2D device can find a desired file - selected at random according to a request distribution - within its radio range. We explore this problem quantitatively in this paper by considering different spatial content models and deriving, optimizing and comparing the hit probabilities for each of them.

Content caching has received significant attention as a means of improving the throughput and latency of networks without requiring additional bandwidth or other technological improvements. A practical use case is video, which will consume nearly $80 \%$ of all wireless data by 2021 [6]. Video caching is perfectly suited to D2D networks for offloading traffic from cellular networks.

\section{A. Related Work and Motivation}

Research to date on content caching has been mainly focused on two different perspectives. On one hand, researchers have attempted to understand the fundamental limits of caching gain. The gain offered by local caching and broadcasting is characterized in the landmark paper [7]. Although this work does not deal with D2D communications and the caches cannot cooperate, it provides the first attempt to characterize the gain offered by local caching. Scaling of the number of active D2D links and optimal collaboration distance with D2D caching are studied in [8], [9]. Combining random independent caching with short-range D2D communications can significantly improve the throughput [10]. Capacity scaling laws in wireless ad hoc networks are investigated in [11], featuring short link distances, and cooperative schemes for order optimal throughput scaling is proposed in [12]. Capacity scaling laws for single [10], [7] and multi-hop caching networks [13] are also investigated. Physical layer caching is studied in [14] to mitigate the interference, and in [15] to achieve linear capacity scaling. Finite-length analysis of random caching schemes that achieve multiplicative caching gain is presented in [16], [17].

Alternatively, as in the current paper, there are several studies focusing on decentralized caching algorithms that have optimized the caching distribution to maximize the cache hit probability, using deterministic or random caching as in [18], [8] given a base station (BS)user topology. FemtoCaching replaces backhaul capacity with storage capacity at the small cell access points, i.e., helpers, and the optimum way of assigning files to the helpers is analyzed in [19] to minimize the delay. There are also geographic placement models focusing on finding the 
cache locally such as [4], in which the cache hit probability is maximized for SINR, Boolean and overlaid network coverage models, and [20], in which the density of successful receptions is maximized using probabilistic placement. Although most of these strategies suggest that the caching distribution should be skewed towards the most popular content and exploit the diversity of content, and it is not usually optimal to cache just the most popular files, as pointed out in [8], [9]. Further, as the current paper will show, unlike the probabilistic policies, where the files are independently placed in the cache memories of different nodes according to the same distribution [4], [21], and [20]; it is not usually optimal to cache files independently. For larger transmission range and higher network density, we will quantify and see that the hit-maximizing caching strategy can be increasingly skewed away from independently caching the popular files.

Recent studies also address problems at the intersection of the hit probability and the spatial throughput. The spatial throughput in D2D networks is optimized by suitably adjusting the proportion of active devices in [22]. Exploiting stochastic geometry, a Poisson cluster model is proposed in [23] and the area spectral efficiency is maximized assuming that the desired content is available inside the same cluster as the typical device. Some of the existing work focuses on mitigating excessive interference to maximize the throughput or capacity, as in [15], [3], [14]. Employing probabilistic caching, cache-aided throughput, which measures the density of successfully served requests by local device caches, is investigated in [5]. The optimal caching probabilities obtained by cache-aided throughput optimization provide throughput gain, particularly in dense user environments compared with the cache-hit-optimal case.

Challenges for the adoption of caching for wireless access networks also include making timely estimates of varying content popularity [24]. Cache update algorithms exploiting the temporal locality of the content have been well studied [25]. Inspired from the Least Recently Used (LRU) replacement principle, a multi-coverage caching policy at the edge-nodes is proposed in [26], where caches are updated in a way that provides content diversity to users who are covered by more than one node. Although [26] combines the temporal and spatial aspects of caching and approaches the performance of centralized policies, it is restricted to the LRU principle.

\section{B. Contributions and A High Level Summary}

We consider a spatial D2D network setting in which the D2D user locations are modeled by a Poisson point process (PPP), and users have limited communication range and finite storage. The D2D users are served by each other if the desired content is cached at a user within its 
radio range: this is called a hit. Otherwise, they are served by the cellular network base station, which is what D2D communication aims to avoid.

We concentrate exclusively on the content placement phase in the above setting in order to maximize the cache hit probability via exploiting the spatial diversity. We do not focus on the transmission phase that incorporates the path loss, fading or interference. The coverage process of the proposed scheme is represented by a Boolean model (BM). The BM is tractable for the noise-limited regime [4], where the interference is small compared to the noise. The coverage area of the BM is determined by a fixed communication radius, as will be detailed in Sect. II.

Spatial caching, pairwise interactions and Matérn hard-core-inspired placement. We introduce a spatial content distribution model for a D2D network, and describe the cache hit probability maximization problem in Sect. III. Our aim is to extend the independent content placement strategy, also known as geographic content placement (GCP) [4], where there is no spatial correlation in placement, which we discuss in Sect. III Exploiting the Matérn hard-core $(\mathrm{MHC})$ models, we propose novel spatially correlated cache placement strategies that enable spatial diversity to maximize the D2D cache hit probability. In Sect. IV] we detail the MHC placement and analyze two different MHC placement strategies: (i) HCP-A that can provide a significantly higher cache hit probability than the GCP scheme in the small cache size regime and (ii) HCP-B that has a higher hit probability than GCP for short ranges.

The key differences from the independent placement model. The device locations follow the PPP distribution, which provides a random deployment instead of a fixed pattern, and hence it is possible to have cache clusters and isolated caches [27], and the content placement distribution is optimized accordingly. Unlike the independent placement model, where the cache placement distribution is independent and identically distributed (i.i.d.) over the spatial domain, the MHC model captures the pairwise interactions between the D2D nodes and yields a negatively correlated placement. The caches storing a particular file are never closer to each other than some given distance, called the exclusion radius, meaning that neighboring users are not likely to cache redundant content. Hence, the radius of exclusion plays the role of a substitute for caching probability.

Comparisons and design insights. Sect. $\mathrm{V}$ provides a simulation study to compare the performance between the different content placement strategies. Independent content placement does not exploit D2D interactions at the network level, and our results show that geographic placement should exploit locality of content, which is possible through negatively correlated 
placement. For short range communication and small cache sizes, HCP is preferred, and when the network intensity is fixed, the cache hit rate gain of the HCP model over the GCP and caching most popular content schemes can reach up to $37 \%$ and $50 \%$, respectively when the communication range is improved, as demonstrated in Sect. V.

\section{System Model And Problem Formulation}

The locations of the D2D users are modeled by a PPP $\Phi$ with density $\lambda_{t}$ as in [28]. We assume that there are $M$ total files in the network, where all files have the same size, and each user has the same cache size $N<M$. Depending on its cache state, each user makes requests for new files based on a general popularity distribution over the set of the files. The popularity of such requests is modeled by the Zipf distribution, which has probability mass function (pmf) $p_{r}(n)=\frac{1}{n^{\gamma_{r}}} / \sum_{m=1}^{M} \frac{1}{m^{\gamma_{r}}}$, for $n=1, \ldots, M$, where $\gamma_{r}$ is the Zipf exponent that determines the skewness of the distribution. The demand profile is Independent Reference Model (IRM), i.e., the standard synthetic traffic model in which the request distribution does not change over time [29]. Our objective is to maximize the average cache hit probability performance of the proposed caching model. Therefore, it is sufficient to consider a snapshot of the network 1 , in which the $\mathrm{D} 2 \mathrm{D}$ user realization is given and requests are i.i.d. over the space. We devise a spatially correlated probabilistic placement policy, in which the D2D caches are loaded in a distributed manner via additional marks attached to them without accounting for any cost, in a timescale that is much shorter than the time over which the locations are predicted, as will be detailed in Sect. IV.

Consider a given realization $\phi=\left\{x_{i}\right\} \subset \mathbb{R}^{2}$ of the PPP $\Phi$. The coverage process of the proposed model can be represented by a Boolean model (BM) [30, Ch. 3]. Specifically, given a transmit power $P$, if we only consider path loss (with exponent $\alpha$ ), no fading and no interference, the received signal at the boundary should be larger than a threshold to guarantee coverage, i.e., $\operatorname{Pr}^{-\alpha} \geq T$, yielding $r \leq \mathrm{R}_{\mathrm{D} 2 \mathrm{D}}=(P / T)^{1 / \alpha}$. Hence, D2D users can only communicate within a finite range, which we call the $\mathrm{D} 2 \mathrm{D}$ radius, denoted by $\mathrm{R}_{\mathrm{D} 2 \mathrm{D}}$. A file request is fulfilled by the D2D users within $R_{D 2 D}$ if one has the file; else the D2D user is served by a BS.

\footnotetext{
${ }^{1}$ Extension of the model to also incorporate the temporal correlation of real traffic traces can be done by exploiting models like the Shot-Noise Model (SNM). This overcomes the limitations of the IRM by explicitly accounting for the temporal locality in requests for contents [29]. However, in that case, the problem under study will have an additional dimension to optimize over, and to do so, online learning algorithms should be developed to both learn the demand and optimize the spatial placement. The study of the temporal dynamics of the request distribution and the content transmission phase is left as future work.
} 
The BM is driven by a PPP on $\mathbb{R}^{2}$ in which the marks are deterministic (constant), $\tilde{\Phi}=$ $\sum_{i} \delta_{\left(x_{i}, B_{i}\left(\mathrm{R}_{\mathrm{D} 2 \mathrm{D}}\right)\right)}$, whose points $x_{i}$ 's denote the germs, and on disc-shaped grains $B_{i}\left(\mathrm{R}_{\mathrm{D} 2 \mathrm{D}}\right)-\mathrm{a}$ closed ball of fixed radius $\mathrm{R}_{\mathrm{D} 2 \mathrm{D}}$ centered at $x_{i}$ - that model the coverage regions of germs. The BM is a tractable model for the noise-limited regime [4]. The coverage process of the D2D transmitters driven by the $\mathrm{BM}$ is given by the union $V_{\mathrm{BM}}=\bigcup_{i}\left(x_{i}+B_{0}\left(\mathrm{R}_{\mathrm{D} 2 \mathrm{D}}\right)\right)$ [30, Ch. 3]. For the interference-limited regime, there is no notion of communication radius, and the analysis of the coverage becomes more involved. SINR coverage models as in [4] can be exploited to determine the distribution of the coverage number, i.e., the number of D2D users covering the typical receiver. However, this is beyond the scope of the current paper.

To characterize the successful transmission probability, one needs to know the number of users that a typical node can connect to, i.e., the coverage number. Exploiting the properties of the PPP, the distribution of the number of transmitters covering the typical receiver is given by $\mathcal{N}_{P} \sim$ Poisson $\left(\lambda_{\mathrm{t}} \pi \mathrm{R}_{\mathrm{D} 2 \mathrm{D}}^{2}\right)$. Therefore,

$$
\mathbb{P}\left(\mathcal{N}_{P}=k\right)=e^{-\lambda_{\mathrm{t}} \pi \mathrm{R}_{\mathrm{D} 2 \mathrm{D}}^{2}} \frac{\left(\lambda_{\mathrm{t}} \pi \mathrm{R}_{\mathrm{D} 2 \mathrm{D}}^{2}\right)^{k}}{k !}, \quad k \geq 0 .
$$

\section{A. Cache Hit Probability}

Assume that the cache placement at the D2D users is done in a dependent manner. Given $\mathcal{N}_{P}=k$ transmitters cover the typical receiver, let $Y_{(m, i)}$ be the indicator random variable that takes the value 1 if file $m$ is available in the cache located at $x_{i} \in \phi$ and 0 otherwise. Thus, the caching probability of file $m$ in cache $i$ is given by $\mathrm{p}_{\mathrm{c}, \Pi}\left(m, x_{i}\right)=\mathbb{P}\left(Y_{(m, i)}=1\right)$. Optimal content placement is a binary problem where the cache placement constraint $\sum_{m=1}^{M} Y_{(m, i)} \leq N$ is satisfied for all $x_{i} \in \phi$, i.e., $Y_{(m, i)}$ 's are inherently dependent. However, the original problem is combinatorial and is NP-hard [19]. For tractability reasons, we take the expectation of this

relation and obtain our relaxed cache placement constraint: $\sum_{m=1}^{M} \mathrm{p}_{\mathrm{c}, \Pi}\left(m, x_{i}\right) \leq N$. Later, we show there are feasible solutions to the relaxed problem filling up all the cache slots.

The maximum average total cache hit probability, i.e., the probability that the typical user finds the content in one of the D2D users it is covered by, for a content placement strategy $\Pi$ can be evaluated by solving the following optimization formulation:

$$
\begin{aligned}
\max _{\mathrm{p}_{\mathrm{c}, \Pi}} & \mathrm{P}_{\mathrm{Hit}, \Pi} \\
\text { s.t. } & \sum_{m=1}^{M} \mathrm{p}_{\mathrm{c}, \Pi}\left(m, x_{i}\right) \leq N, \quad x_{i} \in \Phi,
\end{aligned}
$$


where the hit probability is given by the following expression:

$$
\mathrm{P}_{\mathrm{Hit}, \Pi}=1-\sum_{m=1}^{M} p_{r}(m) \sum_{k=0}^{\infty} \mathbb{P}\left(\mathcal{N}_{\Pi}=k\right) \mathrm{P}_{\mathrm{Miss}, \Pi}(m, k),
$$

where $\mathbb{P}\left(\mathcal{N}_{\Pi}=k\right)$ is the probability that $k$ transmitters (caches) cover the typical receiver, and $\mathrm{P}_{\text {Miss, } \Pi}(m, k)$ is the probability that $k$ caches cover a receiver, and none has file $m$.

We propose different strategies to serve the D2D requests that maximize the cache hit probability. Assuming a transmitter receives one request at a time and multiple transmitters can potentially serve a request, the selection of an active transmitter depends on the caching strategy. A summary of the symbol definitions and important network parameters are given in Table II.

\section{B. Repulsive Content Placement Design}

Optimizing the marginal distribution for content caching by decoupling the caches of D2D users in a spatial network scenario is not sufficient to optimize the joint performance of the caching. The performance can be improved by developing spatially correlated content placement strategies that exploit the spatial distribution of the D2D nodes, as we propose in this paper.

Negatively correlated spatial placement corresponds to a distance-dependent thinning of the transmitter process so that neighboring users are less likely to have matching contents. This kind of approach is promising from an average cache hit rate optimization perspective. Therefore, we mainly focus on negatively dependent or repulsive content placement strategies.

We next define negative dependence for a collection of random variables.

Definition 1. Random variables $Y_{1}, \ldots, Y_{k}, k \geq 2$, are said to be negatively dependent, if for any numbers $y_{1}, \ldots, y_{k} \in \mathbb{R}$, we have that [31]

$$
\mathbb{P}\left(\bigcap_{i=1}^{k} Y_{i} \leq y_{i}\right) \leq \prod_{i=1}^{k} \mathbb{P}\left(Y_{i} \leq y_{i}\right), \quad \mathbb{P}\left(\bigcap_{i=1}^{k} Y_{i}>y_{i}\right) \leq \prod_{i=1}^{k} \mathbb{P}\left(Y_{i}>y_{i}\right)
$$

Next, in Prop. 1, we state the benefit of negatively correlated placement, which is the basis of future spatially correlated policies including our proposed policy in the current paper.

Proposition 1. Negatively dependent content placement provides a higher average cache hit probability than the independent placement strategies.

Proof. See Appendix A. 


\begin{tabular}{|c|c|}
\hline Symbol & Definition \\
\hline \multicolumn{2}{|l|}{ General System Model Parameters } \\
\hline Baseline PPP with transmitter density $\lambda_{t}$; a realization of the PPP & $\Phi ; \phi=\left\{x_{i}\right\} \subset \mathbb{R}^{2}$ \\
\hline D2D communication radius; closed ball centered at $x_{i}$ with radius $\mathrm{R}_{\mathrm{D} 2 \mathrm{D}}$ & $\mathrm{R}_{\mathrm{D} 2 \mathrm{D}} ; B_{i}\left(\mathrm{R}_{\mathrm{D} 2 \mathrm{D}}\right)$ \\
\hline The coverage process of the $\mathrm{D} 2 \mathrm{D}$ transmitters driven by the $\mathrm{BM}$ & $V_{\mathrm{BM}}=\bigcup_{i}\left(x_{i}+B_{0}\left(\mathrm{R}_{\mathrm{D} 2 \mathrm{D}}\right)\right)$ \\
\hline File request distribution; Zipf request exponent & $p_{r}(\cdot) \sim \operatorname{Zipf}\left(\gamma_{\mathrm{r}}\right) ; \gamma_{r}$ \\
\hline Caching probability of file $m$ in cache $i$ & $\mathrm{p}_{\mathrm{c}, \Pi}\left(m, x_{i}\right)$ \\
\hline Density of receivers; density of D2D users & $\lambda_{\mathrm{r}} ; \lambda_{\mathrm{t}}$ \\
\hline Number of D2D users covering a receiver under strategy $\Pi$ & $\mathcal{N}_{\Pi}$ \\
\hline Hit probability for placement strategy $\Pi$ & $\mathrm{P}_{\mathrm{Hit}, \Pi}$ \\
\hline \multicolumn{2}{|l|}{ Miss probability of file $m$ given $k$ users cover the } \\
\hline typical receiver for placement strategy $\Pi$ & $\mathrm{P}_{\text {Miss }, \Pi}(m, k)$ \\
\hline Total number of files; cache size & $M ; N<M$ \\
\hline \multicolumn{2}{|l|}{ Independent Content Placement Design } \\
\hline The caching distribution for independent placement & $\mathrm{p}_{\mathrm{c}, \mathrm{I}}(m)$ \\
\hline The caching distribution for geographic content placement (GCP) in [4] & $\mathrm{p}_{\mathrm{c}, \mathrm{G}}(m)$ \\
\hline The caching distribution for caching most popular content (MPC) & $\mathrm{p}_{\mathrm{c}, \mathrm{MPC}}(m)=1_{m \leq N}$ \\
\hline \multicolumn{2}{|l|}{ Hard-Core Content Placement (HCP) Design } \\
\hline HCP-A model constructed from the underlying PPP $\Phi$ & $\Phi_{M}$ \\
\hline Exclusion radius of file $m$ for the HCP-A model & $r_{m}$ \\
\hline The density of the HCP-A model for file $m$ & $\lambda_{\mathrm{HCP}-\mathrm{A}}(m)$ \\
\hline The number of neighboring transmitters in $B_{0}\left(r_{m}\right)$ & $C_{m} \sim \operatorname{Poisson}\left(\bar{C}_{m}\right), \bar{C}_{m}=\lambda_{\mathrm{t}} \pi r_{m}^{2}$ \\
\hline The number of transmitters containing file $m$ in $B_{0}\left(\mathrm{R}_{\mathrm{D} 2 \mathrm{D}}\right)$ & $\tilde{C}_{m}$ \\
\hline $2 k$ dimensional bounded region $[0, D]^{2 k}$ & $\mathcal{D}^{k}=[0, D]^{2 k}$ \\
\hline The cache miss region given there exists $k$ nodes & $\mathcal{V}^{k}=[0, D]^{2 k} \backslash\left[0, \mathrm{R}_{\mathrm{D} 2 \mathrm{D}}\right]^{2 k}$ \\
\hline Second-order product density for file $m$ & $\rho_{m}^{(2)}(r)$ \\
\hline
\end{tabular}

TABLE I: Notation.

In the remainder of this paper, we first discuss the independent content placement model in Sect. III, which is a special case of the geographic content placement (GCP) problem using the Boolean model first proposed in [4].

We then ask the following question: Given the coverage number $k$ and file $m$, how large cache hit rates can we achieve, i.e., how small can $\mathrm{P}_{\mathrm{Miss}, \mathrm{N}}(m, k) \leq \mathbb{P}\left(Y_{m}=0\right)^{k}$ get for a spatial content placement setting, or what is the best negatively dependent content placement strategy? To answer that, we consider a negatively dependent content placement strategy inspired from the Matérn hard-core processes MHC (type II), which we call as the hard-core content placement (HCP). We detail the HCP model in Sect. IV. 


\section{IndePendent Content Placement Design}

Independent cache placement design is the baseline model where the files are cached at the D2D users identically and independently of each other. Let $\mathrm{p}_{\mathrm{c}, \mathrm{I}}(m)=p_{c}\left(m, x_{i}\right)=\mathbb{P}\left(Y_{m}=1\right)$ be the caching probability of file $m$ in any cache, which is the same at all points $x_{i} \in \phi$.

The maximum average total cache hit probability, i.e., the probability that the typical user finds the content in one of the D2D users it is covered by, can be evaluated by solving

$$
\begin{aligned}
\underset{\mathrm{p}_{\mathrm{c}, \mathrm{I}}}{\max } & \mathrm{P}_{\text {Hit }, \mathrm{I}} \\
\text { s.t. } & \sum_{m=1}^{M} \mathrm{p}_{\mathrm{c}, \mathrm{I}}(m) \leq N,
\end{aligned}
$$

and $\mathrm{P}_{\text {Miss,I }}(m, k)=\left(1-\mathrm{p}_{\mathrm{c}, \mathrm{I}}(m)\right)^{k}$, which is related to $\mathrm{P}_{\mathrm{Hit}, \mathrm{I}}$ through the $\mathrm{P}_{\mathrm{Hit}, \Pi}$ expression in (3).

First, we consider the following trivial case of independent placement, which is clearly suboptimal.

Proposition 2. Caching most popular content MPC. The baseline solution is to store the most popular files only. Letting $Y_{m}=1_{m \leq N}$, i.e., $\mathrm{p}_{\mathrm{c}, \mathrm{MPC}}(m)=1_{m \leq N}$, the miss probability is $\mathrm{P}_{\text {Miss,MPC }}(m, k)=1_{N<m \leq M}$ for all $m$ when $k \geq 1$, and $\mathrm{P}_{\mathrm{Miss}, \mathrm{MPC}}(m, k)=1$ when $k=0$. Hence, the average cache hit probability for the $\mathrm{MPC}$ scheme is $\mathrm{P}_{\mathrm{Hit}, \mathrm{MPC}}=\mathbb{P}\left(\mathcal{N}_{\mathrm{MPC}} \geq 1\right) \sum_{m=1}^{N} p_{r}(m)$.

The independent cache design problem in our paper is a special case of the geographic content placement (GCP) problem using the Boolean model as proposed in [4]. The optimal solution of the GCP problem [4] is characterized by Theorem 1.

Theorem 1. Geographic Content Placement (GCP) [4, Theorem 1]. The optimal caching distribution for the independent placement strategy is given as follows

$$
\mathrm{p}_{\mathrm{c}, \mathrm{G}}^{*}(m)=\left\{\begin{array}{l}
1, \quad \mu^{*}<p_{r}(m) \mathbb{P}\left(\mathcal{N}_{P}=1\right) \\
\frac{1}{\lambda_{\mathrm{t}} \pi \mathrm{R}_{\mathrm{D} 2 \mathrm{D}}^{2}} \log \left(\frac{p_{r}(m) \lambda_{\mathrm{t}} \pi \mathrm{R}_{\mathrm{D} 2 \mathrm{D}}^{2}}{\mu^{*}}\right), \quad p_{r}(m) \mathbb{P}\left(\mathcal{N}_{P}=1\right) \leq \mu^{*} \leq p_{r}(m) \mathbb{E}\left[\mathcal{N}_{P}\right] \\
0, \quad \mu^{*}>p_{r}(m) \mathbb{E}\left[\mathcal{N}_{P}\right]
\end{array}\right.
$$

where $\mathbb{P}\left(\mathcal{N}_{P}=1\right)=e^{-\lambda_{\mathrm{t}} \pi \mathrm{R}_{\mathrm{D} 2 \mathrm{D}}^{2}}\left(\lambda_{\mathrm{t}} \pi \mathrm{R}_{\mathrm{D} 2 \mathrm{D}}^{2}\right), \mathbb{E}\left[\mathcal{N}_{P}\right]=\lambda_{\mathrm{t}} \pi \mathrm{R}_{\mathrm{D} 2 \mathrm{D}}^{2}$. The placement probabilities 
satisfy

$$
p_{r}(j) \sum_{m=1}^{M} \mathbb{P}\left(\mathcal{N}_{P}=m\right) m\left(1-\mathrm{p}_{\mathrm{c}, \mathrm{G}}^{*}(j)\right)^{m-1}=\mu^{*}, \quad j \in\{1, \ldots, M\} .
$$

The optimal variable $\mu^{*}$ satisfies the equality $\sum_{m=1}^{M} \mathrm{p}_{\mathrm{c}, \mathrm{G}}^{*}(m)=N$.

Thus, the optimal value of the average cache hit probability for the GCP model is given by

$$
\mathrm{P}_{\mathrm{Hit}, \mathrm{G}}=\sum_{m=1}^{M} p_{r}(m)\left[1-\exp \left(-\lambda_{\mathrm{t}} \mathrm{p}_{\mathrm{c}, \mathrm{G}}^{*}(m) \pi \mathrm{R}_{\mathrm{D} 2 \mathrm{D}}^{2}\right)\right] .
$$

Proof. See [4, Theorem 1]. It follows from the use of the Lagrangian relaxation method. The solution is found numerically using the bisection method.

Throughout the paper we use the terms independent cache placement and GCP interchangeably.

\section{Hard-Core Content Placement Design}

We next consider the hard-core regime, which provides useful insights for the development of spatial content placement for the regime relevant to D2D communications. Matérn's hard-core (MHC) model is a spatial point process whose points are never closer to each other than some given distance. The proposed content placement model is slightly different from the MHC point process model with fixed radius. Instead, for each file type, a circular exclusion region is created around each D2D transmitter such that the exclusion radius is determined by the popularity of the file. This is to prevent all the D2D transmitters located in a circular region from caching a particular file simultaneously.

We provide two different spatially correlated content placement models both inspired from the Matérn hard-core (MHC) (type II): (i) HCP-A which is an optimized placement model to maximize the average total cache hit probability in (2), and (ii) HCP-B which has the same marginal content placement probability as the GCP model in [4], and is sufficient for achieving a higher cache hit probability than the GCP model.

\section{A. Hard-Core Placement Model I (HCP-A)}

We propose a content placement approach to pick a subset of transmitters based on some exclusion by exploiting the spatial properties of MHC (type II) model, which we call HCP-A. This type of MHC model is constructed from the underlying PPP $\Phi$ modeling the locations of the D2D user caches by removing certain nodes of $\Phi$ depending on the positions of the neighboring 
nodes and additional marks attached to those nodes [30, Ch. 2.1]. Each transmitter of the BM $V_{\mathrm{BM}}$ is assigned a uniformly (i.i.d.) distributed mark $U[0,1]$. A node $x \in \Phi$ is selected if it has the lowest mark among all the points in $B_{x}(R)$, given exclusion radius $R$. A realization of the MHC point process $\Phi_{M}$ is illustrated in Fig. 1

The HCP-A placement model is motivated from the MHC model and implemented as follows. For each file type, there is a distinct exclusion radius $\left(r_{m}\right.$ for file $m$ ) instead of having a fixed exclusion radius $R$. Given a realization $\phi$ of the underlying PPP modeling the locations of the transmitters with intensity $\lambda_{\mathrm{t}}$, we sort the file indices in order of decreasing popularity. For given file index $m$ and radius $r_{m}$, we implement the steps $(a)-(d)$ described in Fig. 1 to determine the set of selected transmitters to place file $m$. For the same realization $\phi$, we implement this procedure for all files. Once a cache is selected $N$ times, then it is full, and no more file can be placed even if it is selected. The objective is to determine the file radii to optimize the placement.

Definition 2. Configuration probability. The probability density function (pdf) of the MHC point process $\Phi_{M}$ with exactly $k$ points in a bounded region $\mathcal{D}=[0, D]^{2} \in \mathbb{R}^{2}$ that denotes the set retained caches that contain file $m$ is given by $f: \mathbb{R}^{2 k} \rightarrow[0, \infty)$ [32. Ch. 5.5] so that

$$
f_{m}(\varphi)=\left\{\begin{array}{l}
a_{m}, \text { if } s_{\varphi}\left(r_{m}\right)=0 \\
0, \text { otherwise }
\end{array}\right.
$$

which is also known as the configuration probability, i.e., the probability that the hard-core model $\Phi_{M}$ takes the realization $\varphi$. In the above, $\varphi=\left\{x_{1}, \ldots, x_{k}\right\} \subset \mathcal{D}$ denotes the set of $k$ points, $a_{m}$ is a normalizing constant and $s_{\varphi}(r)$ is the number of inter-point distances in $\varphi$ that are equal or less than $r$. This yields a uniform distribution 2 of a subset of $k$ points with inter-point distances at least $r_{m}$ in $\mathcal{D}$.

We optimize the exclusion radii to maximize the total hit probability. The exclusion radius of a particular file $r_{m}$ depends on the file popularity in the network, transmitter density and the cache size and satisfies $r_{m}<\mathrm{R}_{\mathrm{D} 2 \mathrm{D}}$. Otherwise, once $r_{m}$ exceeds $\mathrm{R}_{\mathrm{D} 2 \mathrm{D}}$, as holes would start to open up in the coverage for that content, the hit probability for file $m$ would suffer. We consider the following cases: (i) if the file is extremely popular, then many transmitters should simultaneously cache the file, yielding a small exclusion radius, and (ii) if the file is not very

\footnotetext{
${ }^{2}$ The pdf of the retained process 8 is a scaled version of the pdf of the PPP $\Phi$ in which there is no point within the exclusion range of the typical cache. This yields a uniform distribution of $k$ points in $\mathcal{D}$, i.e., $f(\varphi)=a$, where $a$ is a normalizing constant.
} 
popular, then fewer (or zero) transmitters would be sufficient for caching the file, yielding a larger exclusion radius. Therefore, intuitively, we expect the exclusion radius to decrease with increasing file popularity. Our analysis also supports this conclusion that the exclusion radius is inversely related to the file popularity, i.e., the most popular files are stored in a high number of caches with higher marginal probabilities unlike the files with low popularity that are stored with lower marginals, with larger exclusion radius.

By the Slivnyak Theorem, the Palm distribution of the PPP $\Phi$ seen from its typical point (cache) located at 0 corresponds to the law of $\Phi \cup\{0\}$ under the original distribution [30, Ch. 1.4]. Since the typical node (which is at the origin) of $\Phi$ has $C_{m}$ neighbors distributed as $C_{m} \sim$ Poisson $\left(\bar{C}_{m}\right)$ with $\bar{C}_{m}=\lambda_{\mathrm{t}} \pi r_{m}^{2}$, given the exclusion radius $r_{m}$ for file $m$ of the HCP-A model, and the file may be placed at most at only one cache within this circular region. Hence, the probability of a typical D2D transmitter to get the minimum mark in its neighborhood to qualify to cache file $m$, equivalently, the caching probability of file $m$ at a typical transmitter is

$$
\mathrm{p}_{\mathrm{c}, \mathrm{HCP}-\mathrm{A}}(m)=\mathbb{E}\left[\frac{1}{1+C_{m}}\right]=\frac{1-\exp \left(-\bar{C}_{m}\right)}{\bar{C}_{m}} .
$$

From (9), we can easily observe that there is a one-to-one relationship between $r_{m}$ and $\mathrm{p}_{\mathrm{c}, \mathrm{HCP}-\mathrm{A}}(m)$. The inverse relationship between $r_{m}$ and $\mathrm{p}_{\mathrm{c}, \mathrm{HCP}-\mathrm{A}}(m)$ can be seen by taking the following limits:

$$
\lim _{r_{m} \rightarrow 0} \mathrm{p}_{\mathrm{c}, \mathrm{HCP}-\mathrm{A}}(m)=1, \quad \lim _{r_{m} \rightarrow \infty} \mathrm{p}_{\mathrm{c}, \mathrm{HCP}-\mathrm{A}}(m)=0,
$$

which implies that the popular files have small $r_{m}$, hence are cached more frequently, and unpopular files have larger exclusion radii, and are stored at fewer locations.

We denote the density of the HCP-A model for file $m$ by

$$
\lambda_{\mathrm{HCP}-\mathrm{A}}(m)=\frac{\left[1-\exp \left(-\bar{C}_{m}\right)\right]}{\pi r_{m}^{2}}=\mathrm{p}_{\mathrm{c}, \mathrm{HCP}-\mathrm{A}}(m) \lambda_{\mathrm{t}} .
$$

From (11) and (9), we can see that the placement probability of file $m$ is the same as the percentage of nodes that cache the same file.

Let $\tilde{C}_{m}$ be the number of transmitters containing file $m$ within a circular region of radius $\mathrm{R}_{\mathrm{D} 2 \mathrm{D}}$. At most one transmitter is allowed to contain a file within the exclusion radius. Therefore, when $r_{m} \geq \mathrm{R}_{\mathrm{D} 2 \mathrm{D}}$, we have $\tilde{C}_{m} \in\{0,1\}$, and when $r_{m}<\mathrm{R}_{\mathrm{D} 2 \mathrm{D}}$, we have $\tilde{C}_{m} \in\{0,1,2, \cdots\}$.

Proposition 3. The MHC placement is a negatively dependent placement technique. 
Proof. See Appendix B,

As the file popularity increases, the exclusion radius gets smaller. Hence, the average number of transmitters within the exclusion region, i.e., $\bar{C}_{m}^{*}$, decreases, and the chance of having at least one transmitter caching that file within $\mathrm{R}_{\mathrm{D} 2 \mathrm{D}}$ increases, i.e., $\mathbb{P}\left(\tilde{C}_{m} \geq 1\right)>\mathbb{P}\left(\tilde{C}_{n} \geq 1\right)$ for $m<n$. This yields a higher $\mathrm{p}_{\mathrm{c}, \mathrm{HCP}-\mathrm{A}}(\cdot)$ for more popular files from (9). If the demand distribution is uniform over the network, then each file has the same caching probability, i.e., $\mathrm{p}_{\mathrm{c}, \mathrm{HCP}-\mathrm{A}}(m)$ is the same for all $m$, yielding the same $r_{m}$ for all $m$, which is intuitive. When the demand distribution is skewed towards the more popular files, then $\lambda_{\text {HCP-A }}(m)$ scales with the request popularity and $r_{m}$ is inversely proportional to $p_{r}(m)$, i.e., less popular files will end up being stored in fewer locations, and popular files will be guaranteed to be available over a larger geographic area, which is intuitive.

In the HCP-A model, using the pdf in (8) that denotes the configuration of the retained transmitters, the miss probability of file $m$ given $k$ users cover a typical receiver is

$$
\mathrm{P}_{\text {Miss }, \mathrm{MA}}(m, k)=\int \cdots \int_{\mathcal{V}^{k}} f_{m}\left(x_{1}, \ldots, x_{k}\right) \mathrm{d} x_{1} \ldots \mathrm{d} x_{k},
$$

where the region $\mathcal{V}^{k}$ characterizes the cache miss region given there exists $k$ D2D nodes, i.e., it is the $2 k$ dimensional region denoted by $\mathcal{V}^{k}=[0, D]^{2 k} \backslash\left[0, \mathrm{R}_{\mathrm{D} 2 \mathrm{D}}\right]^{2 k}$.

The maximum hit probability for the HCP-A model is given by the solution of

$$
\begin{aligned}
\underset{\mathrm{p}_{\mathrm{c}, \mathrm{HCP}-\mathrm{A}}(m)}{\max } & \mathrm{P}_{\text {Hit }, \text { HCP-A }} \\
\text { s.t. } & \sum_{m=1}^{M} \mathrm{p}_{\mathrm{c}, \mathrm{HCP}-\mathrm{A}}(m) \leq N,
\end{aligned}
$$

and $\mathrm{P}_{\text {Miss,MA }}(m, k)$ is given in (12), which is related to $\mathrm{P}_{\mathrm{Hit}, \mathrm{HCP}-\mathrm{A}}$ through the $\mathrm{P}_{\mathrm{Hit}, \Pi}$ expression given in (3) of the original optimization formulation in (2).

Proposition 4. The average cache hit probability for the HCP-A model is

$$
\mathrm{P}_{\text {Hit }, \mathrm{HCP}-\mathrm{A}}=\sum_{m=1}^{M} p_{r}(m) \mathbb{P}\left(\tilde{C}_{m}>0 \mid r_{m}\right),
$$




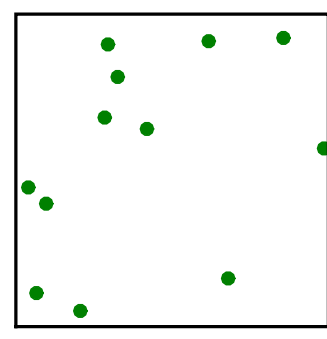

(a)

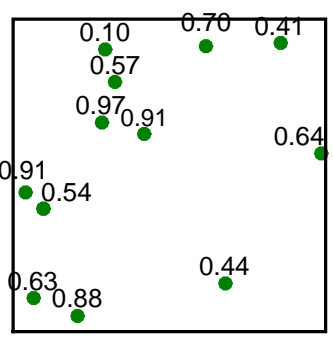

(b)

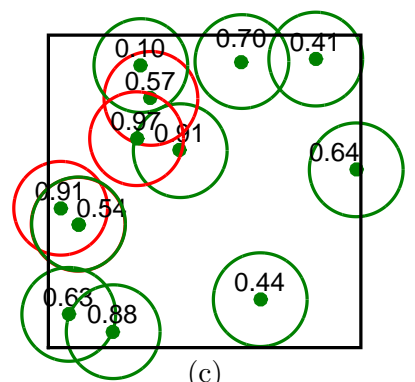

(c)

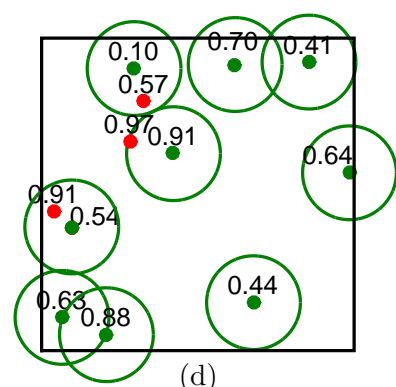

Fig. 1: MHC point process realization for a given exclusion radius $R$ : (a) Begin with a realization of PPP, $\phi$. (b) Associate a uniformly distributed mark $U[0,1]$ to each point of $\phi$ independently. (c) A node $x \in \phi$ is selected if it has the lowest mark inside $B_{x}(R)$. (d) Set of selected points for a given realization of the PPP. We exploit the $\mathrm{MHC}$ model to pick a subset of D2D nodes to cache the files, where there is a distinct exclusion radius for each file, and the exclusion radii are determined by the underlying file popularity distribution.

where the term $\mathbb{P}\left(\tilde{C}_{m}>0 \mid r_{m}\right)$ is essential in determining the cache hit probability and given as

$$
\mathbb{P}\left(\tilde{C}_{m}>0 \mid r_{m}\right)\left\{\begin{array}{l}
\geq 1-\exp \left(-\lambda_{\mathrm{HCP}-\mathrm{A}}(m) \pi \mathrm{R}_{\mathrm{D} 2 \mathrm{D}}^{2}\right), \quad r_{m}<\mathrm{R}_{\mathrm{D} 2 \mathrm{D}}, \\
=\lambda_{\mathrm{HCP}-\mathrm{A}}(m) \pi \mathrm{R}_{\mathrm{D} 2 \mathrm{D}}^{2}, \quad r_{m} \geq \mathrm{R}_{\mathrm{D} 2 \mathrm{D}} .
\end{array}\right.
$$

Proof. See Appendix C.

The optimal solution of the HCP-A model in (13) is characterized by Theorem 2 ,

Theorem 2. Hard-Core Content Placement (HCP). The optimal caching distribution for the HCP model is given as follows

$$
\mathrm{p}_{\mathrm{c}, \mathrm{HCP}-\mathrm{A}}^{*}(m)=\left\{\begin{array}{l}
\lambda_{\mathrm{t}}^{-1} W\left(c p_{r}(m)\right), \quad m \leq \mathrm{m}_{\mathrm{c}} \\
\lambda_{\mathrm{t}}^{-1} c p_{r}(m), \quad m>\mathrm{m}_{\mathrm{c}}
\end{array}\right.
$$

where $W$ is the Lambert function, and $\mathrm{m}_{\mathrm{c}}=\underset{m \in\{1, \cdots, M\}}{\arg \max }\left\{r_{m} \mid r_{m}<\mathrm{R}_{\mathrm{D} 2 \mathrm{D}}\right\}$, and the relation

$$
\sum_{m=1}^{\mathrm{m}_{\mathrm{c}}} W\left(c p_{r}(m)\right)-c p_{r}(m)=N \lambda_{\mathrm{t}}-c
$$

can be used to determined the value of $c$. Hence, we determine $\lambda_{\mathrm{HCP}-\mathrm{A}}^{*}(m)$ and the optimal value of the exclusion radius, i.e., $r_{m}^{*}$, from (17) as a function of the request pmf $p_{r}(m)$, cache size $N$ and the transmitter density $\lambda_{\mathrm{t}}$.

Proof. See Appendix D. 
Consider a ball centered at origin and of radius $D$, i.e., $B_{0}(D)$, with $D \gg \max _{m}\left\{r_{m}\right\}$, let the number of users in $B_{0}(D)$ be Poisson with $\mathbb{P}\left(\mathcal{N}_{P}(D)=k\right)=e^{-\bar{C}_{D} \frac{\left(\bar{C}_{D}\right)^{k}}{k !}}$, where $\bar{C}_{D}=\lambda_{\mathrm{t}} \pi D^{2}$ is the average number of transmitters within $B_{0}(D)$. Due to the limited storage capacity of the caches, the mean total number of files that can be cached in $B_{0}(D)$ is upper bounded by $N \bar{C}_{D}$. To determine the average number of users containing a desired file type in region $B_{0}(D)$, we use the second-order product density of the MHC process $\Phi_{M}$, which is defined next.

Definition 3. Second-order product density [32, Ch. 5.4]. For a stationary point process $\Phi_{M}$, the second-order product density is the joint probability that there are two points of $\Phi_{M}$ at locations $x$ and $y$ in the infinitesimal volumes $d x$ and $d y$, and given by

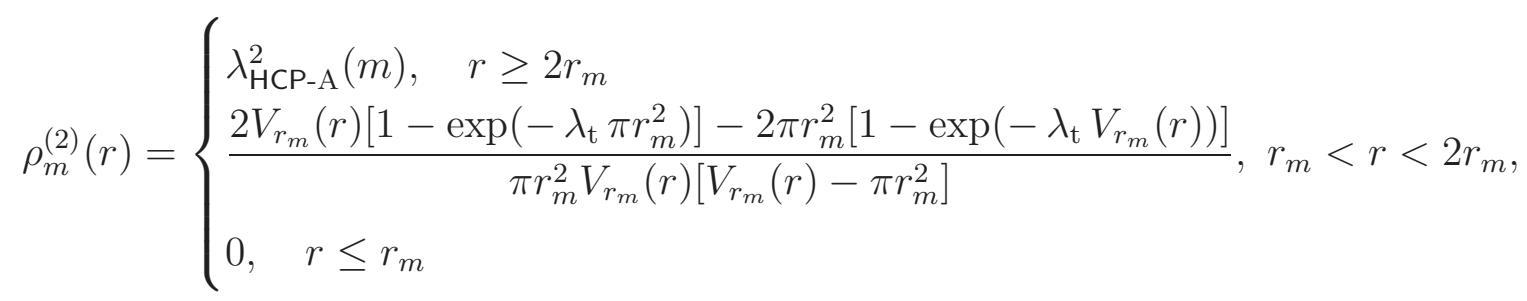

where $\lambda_{t}^{-2} \rho_{m}^{(2)}(r)$ is the two-point Palm probability that two points of $\Phi$ separated by distance $r$ are both retained to store file $m$ [32, Ch. 5.4], and $V_{r_{m}}(r)=2 \pi r_{m}^{2}-2 r_{m}^{2} \cos ^{-1}\left(\frac{r}{2 r_{m}}\right)+$ $r \sqrt{r_{m}^{2}-\frac{r^{2}}{4}}$ is the area of the union of two circles with radius $r_{m}$ and separated by distance $r$. Pairwise correlations between the points separated by $r>r_{m}$ are modeled using the secondorder product density $-\rho_{m}^{(2)}(r)$ for file $m$ - of the $\mathrm{MHC}$ process.

Using the Campbell's theorem [30, Ch. 1.4], we deduce that the average number of transmitters of the stationary point process $\Phi_{M}$-conditioned on there being a point at the origin but not counting it- contained in the ball $B_{0}\left(R_{\mathrm{D} 2 \mathrm{D}}\right)$ is given by

$$
\mathbb{E}^{! \circ}\left[\sum_{x \in \Phi_{M}} 1\left(x \in B_{0}\left(\mathrm{R}_{\mathrm{D} 2 \mathrm{D}}\right)\right)\right]=\lambda_{\mathrm{t}}{ }^{-1} \int_{B_{0}\left(\mathrm{R}_{\mathrm{D} 2 \mathrm{D}}\right)} \rho_{m}^{(2)}(x) d x
$$

An upper bound on the probability that a user requesting file $m$ is covered is given by the following expression:

$$
\begin{aligned}
\mathbb{P}\left(\tilde{C}_{m} \geq 1 \mid r_{m}<\mathrm{R}_{\mathrm{D} 2 \mathrm{D}}\right) & \stackrel{(a)}{\leq} \mathbb{E}\left[\tilde{C}_{m} \mid r_{m}<\mathrm{R}_{\mathrm{D} 2 \mathrm{D}}\right] \\
& \stackrel{(b)}{=} 1-\exp \left(-\lambda_{\mathrm{HCP}-\mathrm{A}}^{*}(m) \pi \mathrm{R}_{\mathrm{D} 2 \mathrm{D}}^{2}\right)+\lambda_{\mathrm{t}}^{-1} \int_{B_{0}\left(\mathrm{R}_{\mathrm{D} 2 \mathrm{D}}\right)} \rho_{m}^{(2)}(x) \mathrm{d} x,
\end{aligned}
$$

where $(a)$ follows from using Markov inequality, and (b) from using (19), to deduce the average 
number of caches that stores file $m$ in $B_{0}\left(\mathrm{R}_{\mathrm{D} 2 \mathrm{D}}\right)$.

Proposition 5. The maximum cache hit probability for the HCP-A model is approximated by the following lower and upper bounds:

$$
\begin{aligned}
& \mathrm{P}_{\mathrm{Hit}, \mathrm{HCP}-\mathrm{A}}^{\mathrm{LB}}=\sum_{m=1}^{\mathrm{m}_{\mathrm{c}}} p_{r}(m)\left[1-e^{-\lambda_{\mathrm{HCP}-\mathrm{A}}^{*}(m) \pi \mathrm{R}_{\mathrm{D} 2 \mathrm{D}}^{2}}\right]+\sum_{m=\mathrm{m}_{\mathrm{c}}+1}^{M} p_{r}(m) \lambda_{\mathrm{HCP}-\mathrm{A}}^{*}(m) \pi \mathrm{R}_{\mathrm{D} 2 \mathrm{D}}^{2}, \\
& \mathrm{P}_{\mathrm{Hit}, \mathrm{HCP}-\mathrm{A}}^{\mathrm{UB}}=\mathrm{P}_{\mathrm{Hit}, \mathrm{HCP}-\mathrm{A}}^{\mathrm{LB}}+\sum_{m=1}^{\mathrm{m}_{\mathrm{c}}} p_{r}(m) \lambda_{\mathrm{t}}^{-1} \int_{r_{m}^{*}}^{\mathrm{R}_{\mathrm{D} 2 \mathrm{D}}} \rho_{m}^{(2)}(x) \mathrm{d} x,
\end{aligned}
$$

where $\bar{C}_{m}^{*}=\lambda_{\mathrm{t}} \pi\left(r_{m}^{*}\right)^{2}$ with each $r_{m}^{*}$ denoting the optimal value of the radius $r_{m}$ for $m=$ $1, \ldots, M$ that maximizes the average cache hit probability, and $\lambda_{\mathrm{HCP}-\mathrm{A}}^{*}(m)$ follows from plugging $r_{m}^{*}$ into $(\underline{11})$.

Proof. See Appendix E.

To compare the performance of the GCP and the HCP models in terms of their average cache hit probabilities, we next consider an example.

Example 1. Cache hit rate comparison for GCP and HCP. Consider a simple caching scenario with $M=2$ files and a cache size of $N=1$, and the request distribution satisfies $p_{r}(1)=2 / 3$ and $p_{r}(2)=1 / 3$. Let $\lambda_{\mathrm{t}} \pi=1$ and assume $\mathrm{R}_{\mathrm{D} 2 \mathrm{D}}$ is given.

- In the GCP model, from Theorem 1 given the product $\lambda_{\mathrm{t}} \pi \mathrm{R}_{\mathrm{D} 2 \mathrm{D}}^{2}$, the values of $\mathbb{P}\left(\mathcal{N}_{P}=1\right)$, $\mathbb{E}\left[\mathcal{N}_{P}\right]$ can be computed. Checking the conditions in (5), the optimal value of $\mu$, and $\mathrm{p}_{\mathrm{c}, \mathrm{G}}^{*}(1)$ and $\mathrm{p}_{\mathrm{c}, \mathrm{G}}^{*}(2)$ can be determined. Thus, from (7), the optimal cache hit probability for the $\mathrm{GCP}$ model becomes $\mathrm{P}_{\mathrm{Hit}, \mathrm{G}}^{*}=\sum_{m=1}^{2} p_{r}(m)\left[1-\exp \left(-\mathrm{p}_{\mathrm{c}, \mathrm{G}}^{*}(m) \lambda_{\mathrm{t}} \pi \mathrm{R}_{\mathrm{D} 2 \mathrm{D}}^{2}\right)\right]$.

- In the HCP model, from (11), we have $\lambda_{\mathrm{HCP}-\mathrm{A}}(m)=\frac{\left[1-\exp \left(-\bar{C}_{m}\right)\right]}{\pi r_{m}^{2}}=\mathrm{p}_{\mathrm{c}, \mathrm{HCP}-\mathrm{A}}(m) \lambda_{\mathrm{t}}$ for $m=1,2$. Using the cache constraint, $\sum_{m=1}^{2} \lambda_{\mathrm{HCP}-\mathrm{A}}(m)=\lambda_{\mathrm{t}}$. Thus, from (14), the cache hit probability for the GCP model becomes $\mathrm{P}_{\mathrm{Hit}, \mathrm{HCP}-\mathrm{A}}=2 / 3 \mathbb{P}\left(\tilde{C}_{1}>0 \mid r_{1}\right)+1 / 3 \mathbb{P}\left(\tilde{C}_{2}>0 \mid r_{2}\right)$, where from (15), we compute $\mathbb{P}\left(\tilde{C}_{m}>0 \mid r_{m}\right)$ using the lower bound in Prop. 5 The optimal values $\mathrm{P}_{\mathrm{Hit}, \mathrm{G}}^{*}, \mathrm{P}_{\text {Hit,HCP-A }}^{\mathrm{LB}}$ for different $\mathrm{R}_{\mathrm{D} 2 \mathrm{D}}$ are tabulated in Table III For $\mathrm{R}_{\mathrm{D} 2 \mathrm{D}}$ high, as the lower bound of the $\mathrm{HCP}$ model is very close to $\mathrm{P}_{\mathrm{Hit}, \mathrm{G}}^{*}$, both models perform similarly. However, for small $\mathrm{R}_{\mathrm{D} 2 \mathrm{D}}$, the $\mathrm{HCP}$ model outperforms (with a cache hit rate gain up to $25 \%$ using the lower bound) because it can exploit the spatial diversity.

Ideally, when a cache placement strategy is applied, the files need to be placed at a cache 


\begin{tabular}{|l|c|c|c|c|c|c|}
\hline $\mathrm{R}_{\mathrm{D} 2 \mathrm{D}}$ & $\mu^{*}$ & $\mathrm{p}_{\mathrm{c}, \mathrm{G}}^{*}(1), \mathrm{p}_{\mathrm{c}, \mathrm{G}}^{*}(2)$ & $\mathrm{P}_{\mathrm{Hit}, \mathrm{G}}^{*}$ & $r_{1}^{*}, r_{2}^{*}$ & $\lambda_{\mathrm{HCP}-\mathrm{A}}^{*}(1), \lambda_{\mathrm{HCP}-\mathrm{A}}^{*}(2)$ & $\mathrm{P}_{\mathrm{Hit}, \mathrm{HCP}-\mathrm{A}}^{\mathrm{LB}}$ \\
\hline$\sqrt{0.5}$ & 0.1836 & 1,0 & $\mathbf{0 . 2 6 2 3}$ & $0.7071,1.7117$ & $0.2813,0.0370$ & $\mathbf{0 . 3 1 4 0}$ \\
$\sqrt{0.75}$ & 0.2430 & $0.9621,0.0379$ & $\mathbf{0 . 3 5 2}$ & $0.866,1.4283$ & $0.2428,0.0756$ & $\mathbf{0 . 4 4 0 7}$ \\
1 & .28592 & $0.8466,0.1534$ & $\mathbf{0 . 4 2 8 2}$ & $1,1.257$ & $0.201,0.1174$ & $\mathbf{0 . 5 4 3 8}$ \\
$\sqrt{2}$ & 0.3468 & $0.6733,0.3267$ & $\mathbf{0 . 6 5 3 2}$ & $0.8718,1.4178$ & $0.2411,0.0772$ & $\mathbf{0 . 6 8 1 8}$ \\
$\sqrt{3}$ & 0.3156 & $0.6155,0.3845$ & $\mathbf{0 . 7 8 9 6}$ & $1.0149,1.2410$ & $0.1961,0.1222$ & $\mathbf{0 . 7 8 9 6}$ \\
$\sqrt{10}$ & 0.0318 & $0.5347,0.4653$ & $\mathbf{0 . 9 9 3 6}$ & $1.0909,1.1576$ & $0.1704,0.1479$ & $\mathbf{0 . 9 9 3 6}$ \\
10 & $9.0926 e^{-21}$ & $0.5035,0.4965$ & $\mathbf{1}$ & $1.1225,1.1225$ & $0.1592,0.1592$ & $\mathbf{1}$ \\
\hline
\end{tabular}

TABLE II: Numerical results for Example 1, with $M=2, N=1$ and $p_{r}(1)=2 / 3 p_{r}(2)=1 / 3$, where the results for the HCP model are obtained by optimizing $\mathrm{P}_{\mathrm{Hit}, \mathrm{HCP}-\mathrm{A}}^{\mathrm{LB}}$ in (21) of Proposition 5 .

in a way that all the cache slots are occupied. In the GCP model in [4], authors propose a probabilistic placement policy to fill the caches. However, in the case of HCP-A placement, due to the random assignment of the marks in each cache independently for distinct files, it is not guaranteed that all the caches are full in the HCP-A approach, which causes underutilization of the caches as detailed next.

Proposition 6. Cache underutilization. The HCP placement model causes underutilization of the caches, i.e., on average, the fraction of the D2D nodes of $\Phi$ that contain $N$ distinct files is always less than 1. This can be formally stated as follows:

$$
\frac{1}{N \mathbb{E}\left[\mathcal{N}_{P}\right]} \sum_{m=1}^{M} \mathbb{E}\left[\tilde{C}_{m}\right] \leq 1,
$$

where $\mathbb{E}\left[\mathcal{N}_{P}\right]=\lambda_{\mathrm{t}} \pi \mathrm{R}_{\mathrm{D} 2 \mathrm{D}}^{2}$.

Proof. See Appendix F

The storage size $N$ and the exclusion radius $r_{m}$ have an inverse relationship. As $N$ drops, because it is not possible to cache the files at all the transmitters, the exclusion radius should increase to bring more spatial diversity into the model. From the storage constraint in (13), as $N$ drops, $r_{m}$ increases $\left(r_{m} \rightarrow \infty\right.$ as $\left.N \rightarrow 0\right)$. Hence, a typical receiver won't be able to find its requested files within its range. When $N$ increases sufficiently, $r_{m}$ can be made smaller so that more files can be cached at the same transmitter $\left(r_{m} \rightarrow 0\right.$ as $\left.N \rightarrow \infty\right)$. Hence, the typical receiver will most likely have the requested files within its range.

Proposition 7. A sufficient condition for the HCP-A placement model. The HCP-A performs 
better than the independent placement model (GCP) [4] in terms of hit probability if the following condition is satisfied:

$$
\lambda_{\mathrm{HCP}-\mathrm{A}}(m) \geq\left\{\begin{array}{l}
\lambda_{\mathrm{t}} \mathrm{p}_{\mathrm{c}, \mathrm{G}}^{*}(m), \quad r_{m}<\mathrm{R}_{\mathrm{D} 2 \mathrm{D}}, \\
\frac{1-\exp \left(-\lambda_{\mathrm{t}} \mathrm{p}_{\mathrm{c}, \mathrm{G}}^{*}(m) \pi \mathrm{R}_{\mathrm{D} 2 \mathrm{D}}^{2}\right)}{\pi \mathrm{R}_{\mathrm{D} 2 \mathrm{D}}^{2}}, \quad r_{m} \geq \mathrm{R}_{\mathrm{D} 2 \mathrm{D}},
\end{array}\right.
$$

where $\mathrm{p}_{\mathrm{c}, \mathrm{G}}^{*}(m)$ is the optimal caching distribution for the GCP.

Proof. See Appendix G.

In the regime where $r_{m}$ is chosen to satisfy the inequality in (23), for all $m$, the HCP-A placement model performs better than independent placement, and the volume fraction occupied by the transmitters caching file $m$, i.e., the proportion of space covered by the union $\bigcup_{x_{i} \in \Phi_{M}}\left(x_{i}+B_{0}\left(\mathrm{R}_{\mathrm{D} 2 \mathrm{D}}\right)\right)$ pertaining to file $m$, is lower bounded by $\frac{\lambda_{\mathrm{HCP}-\mathrm{A}}(m)}{\lambda_{\mathrm{t}}} \geq \frac{1-e^{-\lambda_{\mathrm{t}} \mathrm{P}_{\mathrm{c}, \mathrm{G}}^{*}(m) \pi \mathrm{R}_{\mathrm{D} 2 \mathrm{D}}^{2}}}{\lambda_{\mathrm{t}}}$. When the selection of $\lambda_{\text {HCP-A }}(m)$ does not satisfy (23), the volume fraction pertaining to the caches storing file $m$ is upper bounded by $\frac{\lambda_{H C P-A}(m)}{\lambda_{t}}<\mathrm{p}_{c, G}^{*}(m)$.

From (23), the density parameter $\lambda_{\mathrm{HCP}-\mathrm{A}}(m)$ decreases with $\mathrm{R}_{\mathrm{D} 2 \mathrm{D}}$, hence, the exclusion radius $r_{m}$ increases with $\mathrm{R}_{\mathrm{D} 2 \mathrm{D}}$, which is intuitive because as the number of transmitters within the communication range increases a smaller fraction of them should cache the desired content. The exclusion radius decreases with popularity, i.e., $r_{m}$ decreases as $p_{r}(m)$ increases. It also decreases with $\lambda_{\mathrm{t}}$ and the cache size $N$.

We consider two regimes of caching controlled by the cache size $N$, which determines the optimal cache placement solutions for the independent and HCP-A placement models. The spatial diversity of the content is captured by the optimal placement distribution for given $N$. As $N$ increases, content diversity per cache increases and less spatial diversity is required. Therefore, when $N$ is sufficiently large, independent placement is better than HCP-A placement. For the HCP-A placement model, the exclusion radii decrease with the file popularity. However, for small $N$, a higher exclusion radii are required for all files, which will increase the spatial diversity. Therefore, in the regime where $N$ is small, for sufficiently large $\mathrm{R}_{\mathrm{D} 2 \mathrm{D}}$, HCP-A placement performs better than independent placement (GCP).

We next detail another MHC-based model called HCP-B and provide sufficient conditions for achieving a higher cache hit probability than the GCP model of [4]. 


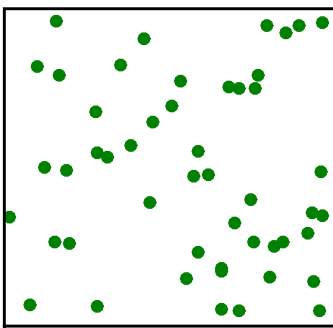

(a) Baseline PPP

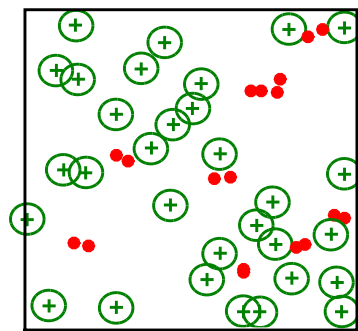

(b) MHC, $R=1$

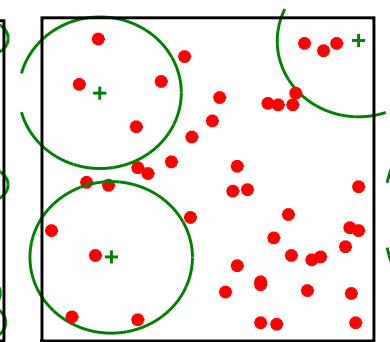

(c) $\mathrm{MHC}, R=5$

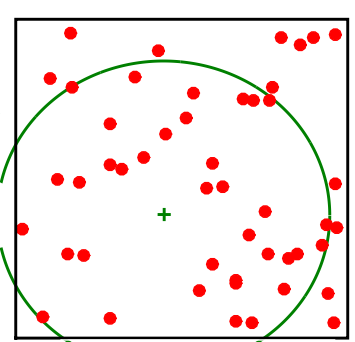

(d) $\mathrm{MHC}, R=10$

Fig. 2: MHC versus the exclusion radii. Each node is associated a uniformly distributed mark $U[0,1]$ independently. Node $x_{i} \in \phi$ is selected if it has the lowest mark in $B_{i}(R)$. Selected nodes are denoted by plus sign. (a) Begin with a realization of PPP, $\phi$. Set of selected points for a given realization of the PPP for an exclusion radius of (b) $R=1$, (c) $R=5$ and (d) $R=10$. As $R$ increases, the intensity of retained nodes decreases.

\section{B. Hard-Core Placement Model II (HCP-B)}

In this section, we propose a new MHC-inspired placement model called HCP-B. We seek a spatially correlated content caching model that improves the performance of the independent placement model of Sect. [II] based on the GCP problem in [4] using the same marginal caching probabilities, i.e., on average the fraction of the users containing a file is equal to its optimal placement probability of the GCP model.

Different from the HCP-A model in Sect. IV-A, where we maximize the average cache hit probability given the finite cache storage constraint, in this section we optimize the exclusion radii using the caching distribution in (5) of the GCP model in Theorem 1, and provide sufficient conditions so that the HCP-B model is at least as good as the GCP scheme of [4].

The critical exclusion radius should be inversely proportional to the popularity of the requests, which is mainly determined by the skewness parameter $\gamma_{r}$. As $\gamma_{r}$ increases, the distribution becomes more skewed and higher variability is observed in the exclusion radii of different files.

In Fig. 2, we illustrate the trend of the MHC process for different exclusion radii. As the exclusion radius $R$ increases, the intensity $\lambda_{\mathrm{MHC}}$ of HCP-B process decreases.

Proposition 8. The exclusion radius for content $m$ for the HCP-B model is given as

$$
r_{m}^{B}=\sqrt{\frac{1}{\lambda_{t} \pi} W\left(-\frac{\exp \left(-1 / \mathrm{p}_{\mathrm{c}, \mathrm{G}}^{*}(m)\right)}{\mathrm{p}_{\mathrm{c}, \mathrm{G}}^{*}(m)}\right)+\frac{1}{\lambda_{t} \pi \mathrm{p}_{\mathrm{c}, \mathrm{G}}^{*}(m)}}, \quad n \in \mathbb{Z},
$$

where $\mathrm{p}_{\mathrm{c}, \mathrm{G}}^{*}(\cdot)$ is the optimal caching distribution for GCP and $W$ is the Lambert function.

Proof. See Appendix $\mathrm{H}$. 
From Prop. 8, given the same marginal caching distributions for the GCP and the HCP-A models, the relation (24) guarantees the HCP-A model to outperform the independent content placement model in terms of the average cache hit rate performance.

Using the second order properties of the hard-core models, the variance of the HCP model is approximated by $\operatorname{Var}_{\mathrm{HCP}-\mathrm{A}} \simeq \lambda_{\mathrm{HCP}-\mathrm{A}}+2 \pi \int_{0}^{\infty}\left(\rho^{(2)}(\mathrm{r})-\lambda_{\text {HCP-A }}^{2}\right) \mathrm{rdr}$ [32, Ch. 4.5]. Hence, using (18) the variance of the MHC model for file $m$ can be approximated as

$$
\operatorname{Var}_{\mathrm{HCP}-\mathrm{A}}(\mathrm{m}) \simeq \lambda_{\mathrm{HCP}-\mathrm{A}}(\mathrm{m})-4 \lambda_{\mathrm{HCP}-\mathrm{A}}(\mathrm{m})\left[1-\exp \left(-\lambda_{\mathrm{t}} \pi \mathrm{r}_{\mathrm{m}}^{2}\right)\right]+2 \pi \int_{\mathrm{r}_{\mathrm{m}}}^{2 \mathrm{r}_{\mathrm{m}}} \rho_{\mathrm{m}}^{(2)}(\mathrm{r}) \mathrm{rdr}
$$

Note that $r_{m}$ decreases, and $\lambda_{\mathrm{HCP}-\mathrm{A}}(m)$ and $\rho_{m}^{(2)}(r)$ increase with popularity. Therefore, we can observe that there is a higher variability for popular files, which means that popular files are placed more randomly than unpopular files, and for unpopular files the placement distribution becomes more regular. This implies that randomized caching is in fact good for popular files, and more deterministic placement techniques are required for unpopular files.

\section{Numerical Comparison of DifFerent Content Placement Models}

We showed that the HCP techniques detailed in Sect. IV yield negatively correlated placement, and can provide a higher cache hit than independent placement (GCP). In this section, we verify our analytical expressions and provide a performance comparison between the GCP of [4], summarized in Sect. III, and the HCP of Sect. IV] by contrasting the average cache hit rates, as discussed in Sect. II. For tractability, in our simulations we assume $M=2$ and $N=1$. The D2D nodes form realizations of a PPP $\Phi$ over the region $[-10,10]^{2}$ with an intensity $\lambda_{t}$ per unit area. We assume there is a typical receiver at the origin which samples a request from the distribution satisfying $p_{r}(1)=2 / 3$ and $p_{r}(2)=1 / 3$. To compute the average cache hit probability performance of different models, we run $10^{5}$ iterations, where at each iteration, we consider a realization $\phi$ of PPP $\Phi$.

Cache hit rate with respect to $\lambda_{t}$. We illustrate the cache hit probability trends of the MPC policy, the GCP model in [4], and the HCP-A and HCP-B placement models together with the bounds for the HCP-A model with respect to the intensity $\lambda_{t}$ for $R_{D 2 D}=10$ in Fig. 3 , It has already been numerically demonstrated in Fig. 3 of [4] that the hit probability of GCP outperforms MPC policy, especially for low SINR thresholds, corresponding to large $R_{\mathrm{D} 2 \mathrm{D}}$ values. Therefore, we use GCP as benchmark for the comparison. The lower and upper bounds for the hit probability of the HCP-A placement in (21) of Prop. 5 is also shown. Compared to the GCP 


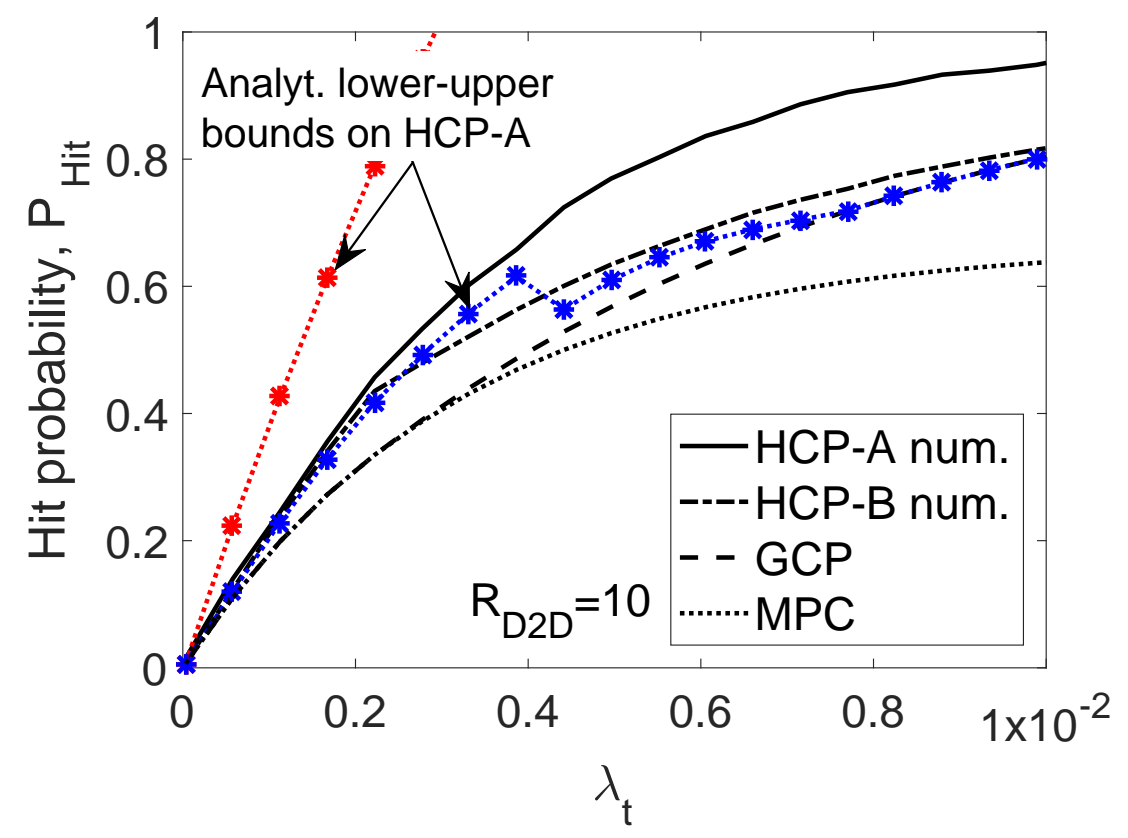

Fig. 3: Maximum cache hit probabilities of the MPC, GCP and HCP model for varying D2D node intensity $\lambda_{t}$.

model in [4], the HCP-A and HCP-B placement models provide higher cache hit probabilities, which we demonstrate next. From Fig. 3, we observe that the average cache hit probability for all cases improves with $\lambda_{t}$, GCP improves with increasing $\lambda_{t}$, and the performance gap between the HCP models and the GCP is higher at high $\lambda_{t}$. The respective cache hit gains of the HCP-B and HCP-A models over GCP can be up to $30 \%$ and $37 \%$, and the gain of HCP-A over MPC is $50 \%$ for this particular example.

Cache hit rate with respect to $R_{D 2 D}$. The numerical comparison for the GCP and the HCP-A models for varying $\mathrm{R}_{\mathrm{D} 2 \mathrm{D}}$ and fixed $\lambda_{t}$ in Example 1 is tabulated in Table III. Now, we illustrate the dependence of the average cache hit probability of different cache placement models on the communication radius $R_{\mathrm{D} 2 \mathrm{D}}$ in Fig. 4. The lower and upper bounds for the hit probability of the HCP-A placement in (21) of Prop. 5is also shown. For high $R_{\mathrm{D} 2 \mathrm{D}}$, both models perform similarly. However, when $\mathrm{R}_{\mathrm{D} 2 \mathrm{D}}$ is small, $\mathrm{HCP}$ performs better because it exploits the spatial diversity of the $\mathrm{D} 2 \mathrm{D}$ caches. For small $\mathrm{R}_{\mathrm{D} 2 \mathrm{D}}$, feasible for the $\mathrm{D} 2 \mathrm{D}$ regime, $\mathrm{MHC}$-inspired approaches are a better alternative 3 .

\footnotetext{
${ }^{3}$ One disadvantage of the HCP-B model is that the excluded files' cache space is not reused, which can be resolved by jointly assigning marks. Therefore, we need to vectorize the marks to jointly determine the set of cached files and to avoid the problems caused by cache underutilization or overuse. The calculation of the cache underutilization or the overuse probability is left as future work.
} 


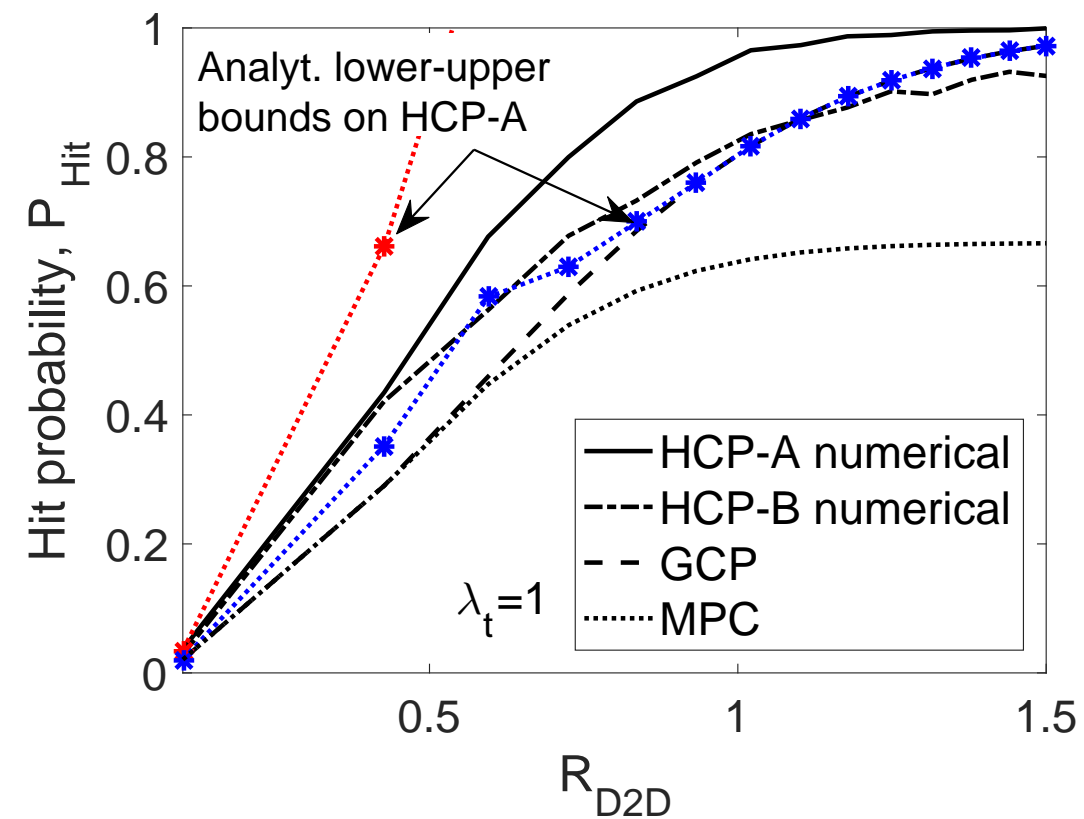

Fig. 4: Maximum cache hit probabilities of the MPC, GCP and HCP models for varying communication radius.

Cache utilization ratio. As discussed in Proposition 6, the HCP placement model causes underutilization of the caches. We numerically investigate the cache utilization ratio for the HCP-A sufficient condition given in Prop. 7, which is shown in Fig. 5, As $R_{\mathrm{D} 2 \mathrm{D}}$ increases, the utilization drops because there will be more D2D caches around the typical receiver and hence, the required number of cache slots decreases. For small $\lambda_{t}$, the values taken by $\lambda_{\text {HCP-A }}(m)$ are small that yields a low utilization ratio when $R_{\mathrm{D} 2 \mathrm{D}}$ is large, which follows from (23). However, the utilization can be improved by jointly determining the values of $\lambda_{\mathrm{HCP}-\mathrm{A}}(m)$ and $\mathrm{R}_{\mathrm{D} 2 \mathrm{D}}$.

Cache size. The performance of the independent and the HCP models is mainly determined by the cache size. Hence, the analysis boils down to finding the critical cache size that determines which model outperforms the other in terms of the hit probability under or above the critical size. In Fig. 6, we show the trend of the optimal exclusion radius $r_{m}$ of the HCP-B model with respect to the caching $\mathrm{pmf}_{\mathrm{c}, \Pi}(m)$. As we expect from (23), the exclusion radius $r_{m}$ decays with the popularity and the cache size $N$. Note that the HCP model compensates the small cache size at the cost of communication radius.

Refinement to soft-core models. The thinning leading to the MHC process can be refined such that higher intensities $\lambda_{\text {HCP-A }}$ are possible [32, Ch. 5.4], at the price of more complicated algorithms [33] and [34]. For refinement of the hard-core models, models based on Gibbs point 


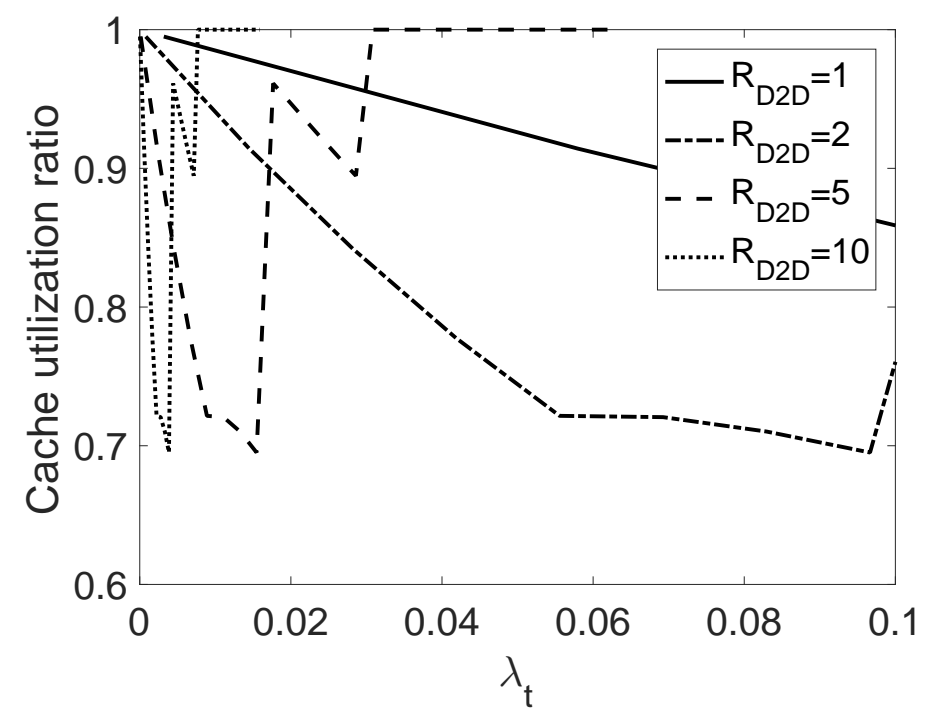

Fig. 5: The cache underutilization (follows from the sufficient condition in Prop. 7).

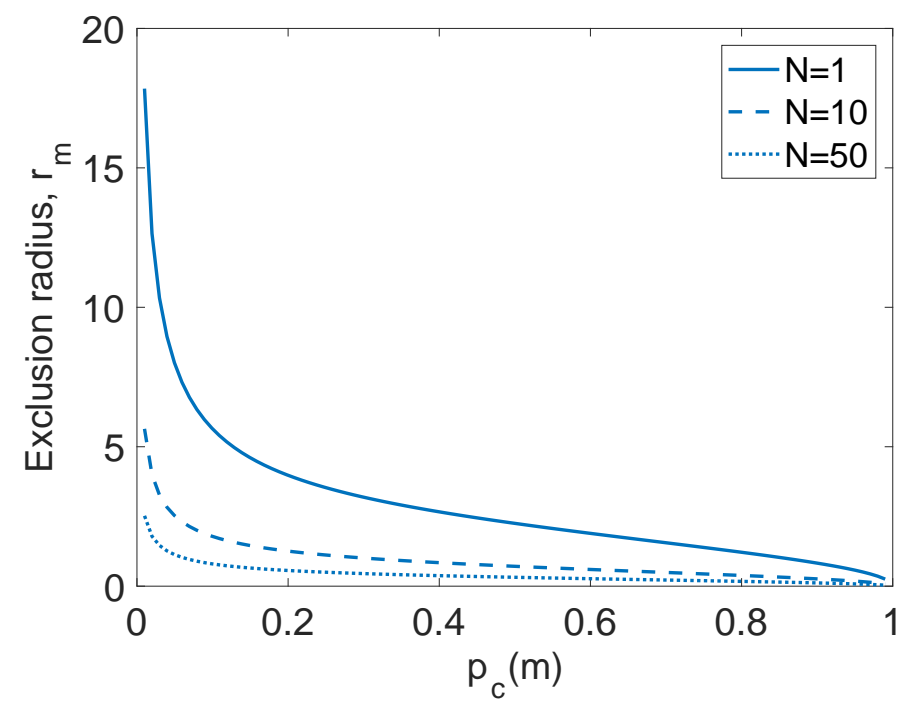

Fig. 6: Characterization of the exclusion radii of $\mathrm{HCP}-\mathrm{B}$ for $N=[1,10,50]$ and $\mathrm{R}_{\mathrm{D} 2 \mathrm{D}}=1$ as a function of $p_{c}(m)$.

processes (GPPs) with repulsive potentials can be developed to generate soft-coret placement models [30, Ch. 18]. The study of soft-core models inspired from GPPs, and the maximum caching gain due to the spreading of content in geographic settings is left as future work.

\footnotetext{
${ }^{4}$ In the case of a soft-core point process, thinning is stronger the closer point pairs of the initial PPP are, but any pair distance still has non-vanishing probability.
} 


\section{CONCLUSIONS}

We proposed spatially correlated content caching models to maximize the hit probability by incorporating hard-core strategies that capture the pairwise interactions to enable spatial diversity.

Our findings on spatial content caching suggest that the following design insights should enable more efficient caching models for D2D-enabled wireless networks:

Repulsive cache placement. Negatively correlated content placement rather than independent placement is required to maximize the cache hit probability. Due to the isotropy of the PPP process, we contemplate a rotation invariant caching model. To satisfy negative spatial correlation, geographical separation of the content within the neighborhood of a typical receiver is required. Thus, in caching protocol design, it is important to incorporate an exclusion region around each cache, such that nodes in this region are not allowed to cache simultaneously. We show that high cache hit rates in a PPP network can be achieved through a MHC-inspired placement model.

Towards soft-core placement models. We analyzed the HCP model, where the exclusions are determined by the hard-core radii. Future studies include more general solutions inspired from the GPP or Ising models capturing the pairwise interactions using soft-core potentials. The shape and scale of the potential should be determined accordingly. The pairwise potential function is promising because it can characterize the spatial and temporal dynamics of the file popularities at different geographic locations adaptively. Hence, the soft-core placement incorporating pairwise correlations can be exploited to improve the cache hit rate. This can can pave the way for the development of spatial cache placement and eviction policies to decide what content to discard, when to discard the content and where (to which neighbor) to relay the content, and provide practical design insights into how to adapt to geographical and temporal changes without compromising the accuracy.

Possible extensions also include hierarchical models for content delivery [25], multi-hop routing to improve the hit probability, distributed scheduling and content caching with bursty arrivals and delay constraints, and smoothing the cellular traffic by minimizing the peak-toaverage traffic ratio with $\mathrm{D} 2 \mathrm{D}$ transmissions.

\section{APPENDIX}

\section{A. Proof of Proposition 1}

For a negatively dependent identical content placement, we can infer that $\mathrm{P}_{\mathrm{Miss}, \mathrm{N}}(m, k) \stackrel{(a)}{\leq}$ $\prod_{i=1}^{k} \mathbb{P}\left(Y_{(m, i)}=0\right) \stackrel{(b)}{=} \mathbb{P}\left(Y_{m}=0\right)^{k}$, where $(a)$ comes from Defn. 1, and $(b)$ is from identical 
content placement assumption. Hence, for a negatively dependent content placement strategy, the hit probability satisfies $\mathrm{P}_{\mathrm{Hit}, \mathrm{N}}=1-\sum_{m=1}^{M} p_{r}(m) \sum_{k=0}^{\infty} \mathbb{P}\left(\mathcal{N}_{P}=k\right) \mathrm{P}_{\mathrm{Miss}, \mathrm{N}}(m, k) \stackrel{(a)}{\geq} 1-$ $\sum_{m=1}^{M} p_{r}(m) \sum_{k=0}^{\infty} \mathbb{P}\left(\mathcal{N}_{P}=k\right) \mathbb{P}\left(Y_{m}=0\right)^{k}$, where the RHS of $(a)$ is the hit probability for independent placement for $\mathrm{p}_{\mathrm{c}, \mathrm{I}}(m)=1-\mathbb{P}\left(Y_{m}=0\right)$.

\section{B. Proof of Proposition 3}

Dropping the file index $m$, let $Y_{i}$ be the indicator random variable that takes the value 1 if file $m$ is available in the cache located at $x_{i} \in \phi$ and 0 otherwise. Given the typical node has $k$ neighbors within its exclusion radius, from (11), the probability that a node $x \in \Phi$ is selected, i.e., has the lowest mark among all the points in $B_{x}(r)$, to cache the file is $\mathbb{P}\left(Y_{i}=1\right)=1 /(k+1)$. For $k>1, \mathbb{P}\left(\bigcap_{i=1}^{k} Y_{i}=0\right)=\mathbb{P}\left(\bigcap_{i=1}^{k} Y_{i}=1\right)=0$ since the probability that all nodes are assigned the same mark values is 0 . Therefore, the following relations in Definition 1 hold:

$$
\mathbb{P}\left(\bigcap_{i=1}^{k} Y_{i}=0\right)<\prod_{i=1}^{k} \mathbb{P}\left(Y_{i}=0\right), \quad \mathbb{P}\left(\bigcap_{i=1}^{k} Y_{i}=1\right)<\prod_{i=1}^{k} \mathbb{P}\left(Y_{i}=1\right),
$$

and the MHC placement satisfies the negative dependence condition in Proposition 1 .

\section{Proof of Proposition 4}

We first consider the case $r_{m} \geq \mathrm{R}_{\mathrm{D} 2 \mathrm{D}}$, where the user can be covered by at most one transmitter that has file $m$. The probability that the user is covered is given by the probability that there exists a transmitter of the HCP-A process of file $m$ at the origin as determined by [30, Ch. 2.1]

$$
\mathbb{P}\left(\tilde{C}_{m}=1 \mid r_{m} \geq \mathrm{R}_{\mathrm{D} 2 \mathrm{D}}\right)=\mathbb{E}\left[\tilde{C}_{m} \mid r_{m} \geq \mathrm{R}_{\mathrm{D} 2 \mathrm{D}}\right]=\lambda_{\mathrm{HCP}-\mathrm{A}}(m) \pi \mathrm{R}_{\mathrm{D} 2 \mathrm{D}}^{2}=\left[1-e^{-\bar{C}_{m}}\right]\left(\frac{\mathrm{R}_{\mathrm{D} 2 \mathrm{D}}}{r_{m}}\right)^{2} .
$$

For the case where $r_{m}<\mathrm{R}_{\mathrm{D} 2 \mathrm{D}}$, we can estimate $\mathbb{P}\left(\tilde{C}_{m} \geq 1 \mid r_{m}<\mathrm{R}_{\mathrm{D} 2 \mathrm{D}}\right)$ using the second-order product density of the MHC model. However, we use a simpler approximation for tractability. The probability that a transmitter is eliminated in the HCP-A with exclusion radius $r_{m}$ is equal to $1-\frac{\lambda_{\mathrm{HCP}-\mathrm{A}}(m)}{\lambda_{\mathrm{t}}}$. For the case of $r_{m}<\mathrm{R}_{\mathrm{D} 2 \mathrm{D}}$, let the number of points in $B\left(r_{m}\right)$ from the original PPP satisfy $\Phi\left(B_{0}\left(R_{\mathrm{D} 2 \mathrm{D}}\right)\right)=k$. Since HCP-A is negatively correlated, from Definition 1, we can exploit the PPP approximation for the MHC in [35] to calculate the following upper bound for the probability that $k$ points are eliminated in HCP-A:

$$
\mathbb{P}\left(k \text { points are eliminated in HCP-A } \Phi_{M} \text { with } r_{m} \mid \mathcal{N}_{P}=k\right) \leq\left(1-\frac{\lambda_{\mathrm{HCP}-\mathrm{A}}(m)}{\lambda_{\mathrm{t}}}\right)^{k} .
$$


Using (27), the void probability of the HCP-A is approximated as

$$
\mathbb{P}\left(\tilde{C}_{m}=0 \mid r_{m}<\mathrm{R}_{\mathrm{D} 2 \mathrm{D}}\right) \leq \sum_{k=0}^{\infty} \mathbb{P}\left(\mathcal{N}_{P}=k\right)\left(1-\frac{\lambda_{\mathrm{HCP}-\mathrm{A}}(m)}{\lambda_{\mathrm{t}}}\right)^{k}=e^{-\lambda_{\mathrm{HCP}-\mathrm{A}}(m) \pi \mathrm{R}_{\mathrm{D} 2 \mathrm{D}}^{2}}
$$

The relations (26) and (28) yield the final result.

\section{Proof of Theorem 2}

Define the Lagrangian to find the solution of (13) as follows:

$$
\begin{aligned}
& \mathcal{M}(\zeta)=\sum_{m=1}^{M} p_{r}(m) \mathbb{P}\left(\tilde{C}_{m}>0 \mid r_{m}\right)+\zeta\left(\sum_{m=1}^{M} \frac{\lambda_{\mathrm{HCP}-\mathrm{A}}(m)}{\lambda_{\mathrm{t}}}-N\right) \\
& \stackrel{(a)}{\approx} \sum_{m=1}^{\mathrm{m}_{\mathrm{c}}} p_{r}(m)\left[1-e^{\left.-\lambda_{\mathrm{HCP}-\mathrm{A}}(m) \pi \mathrm{R}_{\mathrm{D} 2 \mathrm{D}}^{2}\right]}+\sum_{m=\mathrm{m}_{\mathrm{c}}+1}^{M} p_{r}(m) \lambda_{\mathrm{HCP}-\mathrm{A}}(m) \pi \mathrm{R}_{\mathrm{D} 2 \mathrm{D}}^{2}+\zeta\left(\sum_{m=1}^{M} \frac{\lambda_{\mathrm{HCP}-\mathrm{A}}(m)}{\lambda_{\mathrm{t}}}-N\right),\right.
\end{aligned}
$$

where $\mathrm{m}_{\mathrm{c}}=\arg \max _{m \in\{1, \cdots, M\}}\left\{r_{m} \mid r_{m}<\mathrm{R}_{\mathrm{D} 2 \mathrm{D}}\right\}$, and $(a)$ follows from the void probability of the HCP-A for $r_{m}<\mathrm{R}_{\mathrm{D} 2 \mathrm{D}}$ given in (28), and the probability that the user is covered for $r_{m} \geq \mathrm{R}_{\mathrm{D} 2 \mathrm{D}}$ as given in (26). Taking its derivative with respect to $\lambda_{\mathrm{HCP}-\mathrm{A}}(m)$,

$$
\frac{d \mathcal{M}(\zeta)}{d \lambda_{\mathrm{HCP}-\mathrm{A}}(m)}= \begin{cases}p_{r}(m) \pi \mathrm{R}_{\mathrm{D} 2 \mathrm{D}}^{2} e^{-\lambda_{\mathrm{HCP}-\mathrm{A}}(m) \pi \mathrm{R}_{\mathrm{D} 2 \mathrm{D}}^{2}+\zeta \frac{\lambda_{\mathrm{HCP}-\mathrm{A}}(m)}{\lambda_{\mathrm{t}}},} \quad m \leq \mathrm{m}_{\mathrm{C}} \\ p_{r}(m) \pi \mathrm{R}_{\mathrm{D} 2 \mathrm{D}}^{2}+\zeta \frac{\lambda_{\mathrm{HCP}-\mathrm{A}}(m)}{\lambda_{\mathrm{t}}}, \quad m>\mathrm{m}_{\mathrm{C}}\end{cases}
$$

Evaluating this at $\left.\frac{d \mathcal{M}(\zeta)}{d \lambda_{\mathrm{HCP}-\mathrm{A}}(m)}\right|_{\zeta=\zeta^{*}}=0$, we obtain

$$
\zeta^{*}=\left\{\begin{array}{l}
-p_{r}(m) \frac{\lambda_{\mathrm{t}} \pi \mathrm{R}_{\mathrm{D} 2 \mathrm{D}}^{2}}{\lambda_{\mathrm{HCP}-\mathrm{A}}(m)} e^{-\lambda_{\mathrm{HCP}-\mathrm{A}}(m) \pi \mathrm{R}_{\mathrm{D} 2 \mathrm{D}}^{2}}, \quad m \leq \mathrm{m}_{\mathrm{c}} \\
-p_{r}(m) \frac{\lambda_{\mathrm{t}} \pi \mathrm{R}_{\mathrm{D} 2 \mathrm{D}}^{2}}{\lambda_{\mathrm{HCP}-\mathrm{A}}(m)}, \quad m>\mathrm{m}_{\mathrm{c}}
\end{array} .\right.
$$

Note that the optimal solution $\zeta^{*}$ is increasing in the optimal value of $\lambda_{\text {HCP-A }}(m)$, i.e., $\lambda_{\text {HCP-A }}^{*}(m)$. To satisfy this relation, $\lambda_{\mathrm{HCP}-\mathrm{A}}^{*}(m)$ has to satisfy $\lambda_{\mathrm{HCP}-\mathrm{A}}^{*}(m) e^{\lambda_{\mathrm{HCP}-\mathrm{A}}^{*}(m)}=c p_{r}(m)$ for $m \leq \mathrm{m}_{\mathrm{C}}$ and $\lambda_{\mathrm{HCP}-\mathrm{A}}^{*}(m)=c p_{r}(m)$ for $m>\mathrm{m}_{\mathrm{c}}$ for a constant $c$. Using the constraint in (13), we obtain the relation (17) that determines the value of $c$.

\section{E. Proof of Proposition 5}

Incorporating the pdf of the MHC point process with exactly $k$ points given in (8) into the miss probability of the HCP-A model in (12), we derive the cache miss probability for the HCP-A 
model, i.e., the probability that $k$ caches cover a receiver, and none has file $m$, as follows

$$
\mathrm{P}_{\text {Miss }, \mathrm{MA}}(m, k)\left\{\begin{array}{l}
\leq\left(\int_{r_{m}}^{D} \mathrm{~d} x\right)^{k} /\left(\int_{0}^{D} \mathrm{~d} x\right)^{k} \stackrel{(a)}{=}\left(1-\frac{r_{m}^{2}}{D^{2}}\right)^{k}, \quad r_{m}<\mathrm{R}_{\mathrm{D} 2 \mathrm{D}} \\
=\frac{\int_{0}^{1}\left(1-\exp \left(-\bar{C}_{m} t\right)\right) \mathrm{d} t}{\bar{C}_{m}^{-1}}\left(\frac{\mathrm{R}_{\mathrm{D} 2 \mathrm{D}}}{r_{m}}\right)^{2}=\exp \left(-\bar{C}_{m}\right)\left(\frac{\mathrm{R}_{\mathrm{D} 2 \mathrm{D}}}{r_{m}}\right)^{2}, \quad r_{m} \geq \mathrm{R}_{\mathrm{D} 2 \mathrm{D}}
\end{array},\right.
$$

where $\mathcal{V}^{k}$ characterizes the cache miss region given $k$ nodes, and $(a)$ follows from converting the integral into polar coordinates.

Since $\mathrm{P}_{\text {Miss,MA }}(m, k)$ is related to $\mathrm{P}_{\text {Hit,HCP-A }}$ through the $\mathrm{P}_{\mathrm{Hit}, \Pi}$ expression given in (3), the lower bound $\mathrm{P}_{\mathrm{Hit}, \mathrm{HCP}-\mathrm{A}}^{\mathrm{LB}}$ on the maximum cache hit probability for the HCP-A model is given by the final expression in (21), which gives the solution of the the HCP-A hit probability maximization formulation in (13) given the $r_{m}$ values are optimized using the relation (17) of Theorem 2, Similarly, the upper bound P $\mathrm{HB}$ Hit,HCP-A can be found using (20).

\section{F. Proof of Proposition 6}

In the HCP-A model with exclusion radius $r_{m}$, from (11), the average number of nodes in $B_{0}\left(r_{m}\right)$ is given by $1-e^{-\bar{C}_{m}}$. For popular files with $r_{m}<\mathrm{R}_{\mathrm{D} 2 \mathrm{D}}$, the maximum number of nonoverlapping circles with radius $r_{m}$ that can fit inside $B_{0}\left(\mathrm{R}_{\mathrm{D} 2 \mathrm{D}}\right)$ is upper bounded by $\left(\frac{\mathrm{R}_{\mathrm{D} 2 \mathrm{D}}}{r_{m}}\right)^{2}$. Hence, the following inequality is satisfied:

$$
\mathbb{E}\left[\tilde{C}_{m} \mid r_{m}<\mathrm{R}_{\mathrm{D} 2 \mathrm{D}}\right] \leq\left[1-e^{-\bar{C}_{m}}\right]\left(\frac{\mathrm{R}_{\mathrm{D} 2 \mathrm{D}}}{r_{m}}\right)^{2} .
$$

The average number of transmitters that cache all the files in $B_{0}\left(R_{\mathrm{D} 2 \mathrm{D}}\right)$ is given by

$$
\begin{aligned}
\sum_{m=1}^{M} \mathbb{E}\left[\tilde{C}_{m}\right] & =\sum_{m=1}^{\mathrm{m}_{\mathrm{c}}} \mathbb{E}\left[\tilde{C}_{m} \mid r_{m}<\mathrm{R}_{\mathrm{D} 2 \mathrm{D}}\right]+\sum_{m=\mathrm{m}_{\mathrm{c}}+1}^{M} \mathbb{E}\left[\tilde{C}_{m} \mid r_{m} \geq \mathrm{R}_{\mathrm{D} 2 \mathrm{D}}\right] \\
& \stackrel{(a)}{\leq} \sum_{m=1}^{\mathrm{m}_{\mathrm{c}}}\left[1-\exp \left(-\bar{C}_{m}\right)\right]\left(\frac{\mathrm{R}_{\mathrm{D} 2 \mathrm{D}}}{r_{m}}\right)^{2}+\sum_{m=\mathrm{m}_{\mathrm{c}}+1}^{M} \lambda_{\mathrm{HCP}-\mathrm{A}}(m) \pi \mathrm{R}_{\mathrm{D} 2 \mathrm{D}}^{2} \\
& =\sum_{m=1}^{M}\left[1-\exp \left(-\bar{C}_{m}\right)\right]\left(\frac{\mathrm{R}_{\mathrm{D} 2 \mathrm{D}}}{r_{m}}\right)^{2} \stackrel{(b)}{\leq} N \mathbb{E}\left[\mathcal{N}_{P}\right]=N \lambda_{\mathrm{t}} \pi \mathrm{R}_{\mathrm{D} 2 \mathrm{D}}^{2}
\end{aligned}
$$

which results in underutilization of the caches. In the above, the inequality in $(a)$ follows from the inequality (29) for $r_{m}<\mathrm{R}_{\mathrm{D} 2 \mathrm{D}}$, and the equality (26) for $r_{m} \geq \mathrm{R}_{\mathrm{D} 2 \mathrm{D}}$, and $(b)$ from scaling the constraint $\sum_{m=1}^{M} \mathrm{p}_{\mathrm{c}, \mathrm{HCP}-\mathrm{A}}(m) \leq N$ of the hit probability maximization formulation in (13) with $\lambda_{\mathrm{t}} \pi \mathrm{R}_{\mathrm{D} 2 \mathrm{D}}^{2}$ and using the relation (9). Thus, the main reason for the underutilization is the popular files with $r_{m}<\mathrm{R}_{\mathrm{D} 2 \mathrm{D}}$. Despite the underutilization of the caches, from (27), the void probability 
of the very popular files will be insignificant. As the skewness of the Zipf distribution increases, $r_{m}$ for popular $m$ becomes even smaller and in the limit as $p_{r}(1)$ goes to 1 , the value of $r_{1}$ converges to 0 . In that case, since the probability of jointly retaining two nodes separated by any distance will be independent of each other, we observe that (27) will be satisfied with equality. Therefore, the inequality in $(a)$ above that causes the underutilization in (22) will eventually become equality.

\section{G. Proof of Proposition 7}

Using the hit probabilities given in (4) and (13), respectively for the independent and HCP-A content placements, a necessary condition for the HCP-A to perform better than the optimal independent placement model in [4] in terms of hit probability is given by

$\mathrm{P}_{\mathrm{Hit}, \mathrm{HCP}-\mathrm{A}}=\sum_{m=1}^{M} p_{r}(m) \mathbb{P}\left(\tilde{C}_{m} \geq 1 \mid r_{m}\right) \geq \mathrm{P}_{\mathrm{Hit}, \mathrm{G}}=\sum_{m=1}^{M} p_{r}(m)\left[1-\exp \left(-\lambda_{\mathrm{t}} \mathrm{p}_{\mathrm{c}, \mathrm{G}}^{*}(m) \pi \mathrm{R}_{\mathrm{D} 2 \mathrm{D}}^{2}\right)\right]$.

A sufficient condition for (30) to be valid is $\mathbb{P}\left(\tilde{C}_{m} \geq 1 \mid r_{m}\right) \geq 1-\exp \left(-\lambda_{\mathrm{t}} \mathrm{p}_{\mathrm{c}, \mathrm{G}}^{*}(m) \pi \mathrm{R}_{\mathrm{D} 2 \mathrm{D}}^{2}\right)$. For files with very high popularity, from (28) we have

$$
\mathbb{P}\left(\tilde{C}_{m} \geq 1 \mid r_{m}<\mathrm{R}_{\mathrm{D} 2 \mathrm{D}}\right) \geq 1-\exp \left(-\lambda_{\mathrm{HCP}-\mathrm{A}}(m) \pi \mathrm{R}_{\mathrm{D} 2 \mathrm{D}}^{2}\right) \geq 1-\exp \left(-\lambda_{\mathrm{t}} \mathrm{p}_{\mathrm{c}, \mathrm{G}}^{*}(m) \pi \mathrm{R}_{\mathrm{D} 2 \mathrm{D}}^{2}\right)
$$

For files with very low popularity, $r_{m}$ tends to be very high, i.e., $r_{m} \geq \mathrm{R}_{\mathrm{D} 2 \mathrm{D}}$, and from (26),

$$
\mathbb{P}\left(\tilde{C}_{m}=1 \mid r_{m} \geq \mathrm{R}_{\mathrm{D} 2 \mathrm{D}}\right)=\lambda_{\mathrm{HCP}-\mathrm{A}}(m) \pi \mathrm{R}_{\mathrm{D} 2 \mathrm{D}}^{2} \geq 1-\exp \left(-\lambda_{\mathrm{t}} \mathrm{p}_{\mathrm{c}, \mathrm{G}}^{*}(m) \pi \mathrm{R}_{\mathrm{D} 2 \mathrm{D}}^{2}\right)
$$

Solving (31) and (32), the final result is obtained.

The following relation is established from (31) and (32):

$$
\sum_{m=1}^{M} \lambda_{\text {HCP-A }}(m) \geq \sum_{m=1}^{\mathrm{m}_{\mathrm{c}}} \lambda_{\mathrm{t}} \mathrm{p}_{\mathrm{c}, \mathrm{G}}^{*}(m)+\sum_{m=\mathrm{m}_{\mathrm{c}}+1}^{M} \frac{1-\exp \left(-\lambda_{\mathrm{t}} \mathrm{p}_{\mathrm{c}, \mathrm{G}}^{*}(m) \pi \mathrm{R}_{\mathrm{D} 2 \mathrm{D}}^{2}\right)}{\pi \mathrm{R}_{\mathrm{D} 2 \mathrm{D}}^{2}},
$$

where using $1-e^{-x} \leq x$ for $x \geq 0$, the RHS of (33) can be shown to satisfy:

$$
\leq \sum_{m=1}^{\mathrm{m}_{\mathrm{c}}} \lambda_{\mathrm{t}} \mathrm{p}_{\mathrm{c}, \mathrm{G}}^{*}(m)+\sum_{m=\mathrm{m}_{\mathrm{c}}+1}^{M} \lambda_{\mathrm{t}} \mathrm{p}_{\mathrm{c}, \mathrm{G}}^{*}(m)=\lambda_{\mathrm{t}} \sum_{m=1}^{M} \mathrm{p}_{\mathrm{c}, \mathrm{G}}^{*}(m)=N \lambda_{\mathrm{t}}
$$

For a feasible cache placement strategy, we also require that $\sum_{m=1}^{M} \lambda_{\mathrm{HCP}-\mathrm{A}}(m) \leq N \lambda_{\mathrm{t}}$. Hence, it is possible to set $\lambda_{\mathrm{HCP}-\mathrm{A}}(m)$ 's as in (23) and satisfy the feasible placement condition. 


\section{H. Proof of Proposition 8}

In order to compute the exclusion radii $r_{m}^{B}$ for the HCP-B model, we relate the expression (9) for the marginal caching probability of file $m$ of the HCP-A model, which is also true for the HCP-B model, to the optimal placement probability for the GCP model in [4] such that the solution for the exclusion radius $r_{m}^{B}=\sqrt{\bar{C}_{m} /\left(\lambda_{\mathrm{t}} \pi\right)}$ satisfies

$$
\mathrm{p}_{\mathrm{c}, \mathrm{G}}^{*}(m)=\frac{\lambda_{\mathrm{HCP}-\mathrm{A}}(m)}{\lambda_{\mathrm{t}}}=\frac{1-\exp \left(-\bar{C}_{m}\right)}{\bar{C}_{m}}
$$

For such selection of variables $r_{m}^{B}$ 's, the hit probability for the HCP-B model satisfies

$$
\begin{aligned}
\mathrm{P}_{\mathrm{Hit}, \mathrm{HCP}-\mathrm{B}} & =\sum_{m=1}^{\mathrm{m}_{\mathrm{c}}} p_{r}(m)\left[1-e^{-\lambda_{\mathrm{HCP}-\mathrm{A}}(m) \pi \mathrm{R}_{\mathrm{D} 2 \mathrm{D}}^{2}}\right]+\sum_{m=\mathrm{m}_{\mathrm{c}}+1}^{M} p_{r}(m)\left[1-\exp \left(-\bar{C}_{m}\right)\right]\left(\frac{\mathrm{R}_{\mathrm{D} 2 \mathrm{D}}}{r_{m}^{B}}\right)^{2} \\
& =\sum_{m=1}^{\mathrm{m}_{\mathrm{C}}} p_{r}(m)\left[1-e^{\left.-\lambda_{\mathrm{t}} \mathrm{p}_{\mathrm{c}, \mathrm{G}}^{*}(m) \pi \mathrm{R}_{\mathrm{D} 2 \mathrm{D}}^{2}\right]}+\sum_{m=\mathrm{m}_{\mathrm{c}}+1}^{M} p_{r}(m) \lambda_{\mathrm{t}} \mathrm{p}_{\mathrm{c}, \mathrm{G}}^{*}(m) \pi \mathrm{R}_{\mathrm{D} 2 \mathrm{D}}^{2}\right.
\end{aligned}
$$

On the other hand, the hit probability for the GCP model in [4] satisfies (30]). Noting that

$x \geq 1-e^{-x}$ for $x \geq 0$, hence from (7) and (35), we conclude that $\mathrm{P}_{\text {Hit,HCP-B }} \geq \mathrm{P}_{\text {Hit, G. }}$.

\section{REFERENCES}

[1] D. Malak, M. Al-Shalash, and J. G. Andrews, "Optimizing the spatial content caching distribution for device-to-device communications," in Proc., IEEE ISIT, Barcelona, Spain, Jul. 2016, pp. 280-284.

[2] X. Lin, J. G. Andrews, A. Ghosh, and R. Ratasuk, "An overview of 3GPP device-to-device proximity services," IEEE Commun. Mag., vol. 52, no. 4, pp. 40-48, Apr. 2014.

[3] N. Naderializadeh, D. T. Kao, and A. S. Avestimehr, "How to utilize caching to improve spectral efficiency in device-todevice wireless networks," in Proc., Annu. Allerton Conf., Illinois, USA, Oct. 2014.

[4] B. Błaszczyszyn and A. Giovanidis, "Optimal geographic caching in cellular networks," in Proc., IEEE ICC, UK, Jun. 2015, pp. 3358-3363.

[5] Z. Chen, N. Pappas, and M. Kountouris, "Probabilistic caching in wireless D2D networks: Hit optimal vs. throughput optimal," IEEE Communications Letters, vol. 21, no. 3, pp. 584-587, Mar. 2017.

[6] "Cisco visual networking index: Global mobile data traffic forecast update, 2016-2021," white paper, 2017.

[7] M. A. Maddah-Ali and U. Niesen, "Fundamental limits of caching," IEEE Trans. Inf. Theory, vol. 60, no. 5, pp. 2856-67, May 2014.

[8] M. Ji, G. Caire, and A. F. Molisch, "Fundamental limits of caching in wireless D2D networks," IEEE Trans. Inf. Theory, vol. 62, no. 2, pp. 849-869, Feb. 2016.

[9] N. Golrezaei, A. G. Dimakis, and A. F. Molisch, "Scaling behavior for device-to-device communications with distributed caching," IEEE Trans. Inf. Theory, vol. 60, no. 7, pp. 4286-4298, Jul. 2014.

[10] M. Ji, G. Caire, and A. F. Molisch, "The throughput-outage tradeoff of wireless one-hop caching networks," IEEE Trans. Inf. Theory, vol. 61, no. 12, pp. 6833-6859, Dec. 2015.

[11] P. Gupta and P. R. Kumar, "The capacity of wireless networks," IEEE Trans. Inf. Theory, vol. 46, no. 2, pp. 388-404, Mar. 2000.

[12] U. Niesen, P. Gupta, and D. Shah, "On capacity scaling in arbitrary wireless networks," IEEE Trans. Inf. Theory, vol. 55, no. 9, pp. 3959-3982, Sep. 2009. 
[13] S.-W. Jeon, S.-N. Hong, M. Ji, and G. Caire, "Caching in wireless multihop device-to-device networks," in Proc., IEEE ICC, Jun. 2015, pp. 6732-6737.

[14] N. Naderializadeh, M. A. Maddah-Ali, and A. S. Avestimehr, "Fundamental limits of cache-aided interference management," IEEE Trans. Inf. Theory, vol. 63, no. 5, pp. 3092-107, May 2017.

[15] A. Liu and V. K. N. Lau, "How much cache is needed to achieve linear capacity scaling in backhaul-limited dense wireless networks?” IEEE/ACM Trans. Netw., vol. 25, no. 1, pp. 179-88, Feb. 2017.

[16] K. Shanmugam, M. Ji, A. M.Tulino, J. Llorca, and A. G. Dimakis, "Finite length analysis of caching-aided coded multicasting," IEEE Trans. Inf. Theory, vol. 62, no. 10, pp. 5524-37, Oct. 2016.

[17] G. Vettigli, M. Ji, A. M. Tulino, J. Llorca, and P. Festa, "An efficient coded multicasting scheme preserving the multiplicative caching gain,” in Proc., IEEE Infocom Wkshps, Apr., 2015, pp. 251-256.

[18] N. Golrezaei, P. Mansourifard, A. F. Molisch, and A. G. Dimakis, "Base-station assisted device-to-device communications for high-throughput wireless video networks," IEEE Trans. Wireless Comm., vol. 13, no. 7, pp. 3665-3676, Jul. 2014.

[19] K. Shanmugam, N. Golrezaei, A. G. Dimakis, A. F. Molisch, and G. Caire, "FemtoCaching: Wireless content delivery through distributed caching helpers," IEEE Trans. Inf. Theory, vol. 59, no. 12, pp. 8402-13, Dec. 2013.

[20] D. Malak, M. Al-Shalash, and J. G. Andrews, "Optimizing content caching to maximize the density of successful receptions in device-to-device networking," IEEE Trans. Commun., vol. 64, no. 10, pp. 4365-4380, Oct. 2016.

[21] J. Elias and B. Błaszczyszyn, "Optimal geographic caching in cellular networks with linear content coding," arXiv preprint arXiv:1704.08625, Apr. 2017.

[22] B. Błaszczyszyn, P. Keeler, and P. Muhlethaler, "Optimizing spatial throughput in device-to-device networks," arXiv preprint arXiv:1612.09198, Dec. 2016.

[23] M. Afshang, H. S. Dhillon, and P. H. J. Chong, "Modeling and performance analysis of clustered device-to-device networks," IEEE Trans. Wireless Commun., vol. 15, no. 7, pp. 4957-72, Jul. 2016.

[24] M. Leconte, G. Paschos, L. Gkatzikis, M. Draief, S. Vassilaras, and S. Chouvardas, "Placing dynamic content in caches with small population," in Proc., IEEE Infocom, Apr. 2016.

[25] H. Che, Y. Tung, and Z. Wang, "Hierarchical web caching systems: Modeling, design and experimental results," IEEE J. Sel. Areas Commun., vol. 20, no. 7, pp. 1305-1314, Sep. 2002.

[26] A. Giovanidis and A. Avranas, "Spatial multi-LRU caching for wireless networks with coverage overlaps," in Proc., ACM Sigmetrics/IFIP Performance, Antibes, France, Jun. 2016, pp. 403-405.

[27] J. G. Andrews, F. Baccelli, and R. K. Ganti, "A tractable approach to coverage and rate in cellular networks," IEEE Trans. Comm., vol. 59, no. 11, pp. 3122-3134, Nov. 2011.

[28] X. Lin, J. G. Andrews, and A. Ghosh, "Spectrum sharing for device-to-device communication in cellular networks," IEEE Trans. Wireless Comm., vol. 13, no. 12, pp. 6727-6740, Dec. 2014.

[29] S. Traverso, M. Ahmed, M. Garetto, P. Giaccone, E. Leonardi, and S. Niccolini, "Temporal locality in today's content caching: Why it matters and how to model it," ACM SIGCOMM Computer Communication Review, vol. 43, no. 5, pp. 5-12, Nov. 2013.

[30] F. Baccelli and B. Błaszczyszyn, Stochastic Geometry and Wireless Networks. NOW: Found. Trends. Network., 2010.

[31] M. Gerasimov, V. Kruglov, and A. Volodin, "On negatively associated random variables," Lobachevskii Journal of Mathematics, vol. 33, no. 1, pp. 47-55, Jan. 2012.

[32] D. Stoyan, W. Kendall, and J. Mecke, Stochastic Geometry and Its Applications, 2nd ed. John Wiley and Sons, 1996.

[33] J. Møller, M. L. Huber, and R. L. Wolpert, "Perfect simulation and moment properties for the Matérn type III process," Stoch. Process. Appl., vol. 120, no. 11, pp. 2142-58, Nov. 2010.

[34] M. Hörig and C. Redenbach, "The maximum volume hard subset model for Poisson processes: simulation aspects," J. Statist. Comput. Simul., vol. 82, no. 1, pp. 107-121, Jan. 2012.

[35] A. M. Ibrahim, T. ElBatt, and A. El-Keyi, "Coverage probability analysis for wireless networks using repulsive point processes," in Proc., IEEE PIMRC, Sep. 2013, pp. 1002 - 1007. 